\title{
An Eulerian interface sharpening algorithm for compressible two-phase flow: The algebraic THINC approach
}

\author{
Keh-Ming Shyue ${ }^{\mathrm{a}}$, Feng Xiao ${ }^{\mathrm{b}}$ \\ a Institute of Applied Mathematical Sciences, National Taiwan University, Taipei 10617, Taiwan \\ b Department of Energy Sciences, Tokyo Institute of Technology, 4259 Nagatsuta Midori-ku, Yokohama 226-8502, Japan
}

\section{A R T I C L E I N F O}

\section{Article history:}

Received 7 April 2013

Received in revised form 4 December 2013

Accepted 7 March 2014

Available online 18 March 2014

\section{Keywords:}

Compressible two-phase flow

Five-equation model

Interface sharpening

THINC reconstruction

Mie-Grüneisen equation of state

Semi-discrete wave propagation method

\begin{abstract}
A B S T R A C T
We describe a novel interface-sharpening approach for efficient numerical resolution of a compressible homogeneous two-phase flow governed by a quasi-conservative five-equation model of Allaire et al. (2001) [1]. The algorithm uses a semi-discrete wave propagation method to find approximate solution of this model numerically. In the algorithm, in regions near the interfaces where two different fluid components are present within a cell, the THINC (Tangent of Hyperbola for INterface Capturing) scheme is used as a basis for the reconstruction of a sub-grid discontinuity of volume fractions at each cell edge, and it is complemented by a homogeneous-equilibrium-consistent technique that is derived to ensure a consistent modeling of the other interpolated physical variables in the model. In regions away from the interfaces where the flow is single phase, standard reconstruction scheme such as MUSCL or WENO can be used for obtaining high-order interpolated states. These reconstructions are then used as the initial data for Riemann problems, and the resulting fluctuations form the basis for the spatial discretization. Time integration of the algorithm is done by employing a strong stability-preserving Runge-Kutta method. Numerical results are shown for sample problems with the Mie-Grüneisen equation of state for characterizing the materials of interests in both one and two space dimensions that demonstrate the feasibility of the proposed method for interface-sharpening of compressible two-phase flow. To demonstrate the competitiveness of our approach, we have also included results obtained using the anti-diffusion interface sharpening method.
\end{abstract}

(c) 2014 Elsevier Inc. All rights reserved.

\section{Introduction}

Our goal is to describe a novel Eulerian interface-sharpening approach for the efficient numerical resolution of problems with material interfaces arising from inviscid compressible two-phase flow. We consider an unsteady, inviscid, homogeneous two-phase flow that is governed by a five-equation model system of the form

$$
\begin{aligned}
& \frac{\partial}{\partial t}\left(\alpha_{1} \rho_{1}\right)+\nabla \cdot\left(\alpha_{1} \rho_{1} \vec{u}\right)=0 \\
& \frac{\partial}{\partial t}\left(\alpha_{2} \rho_{2}\right)+\nabla \cdot\left(\alpha_{2} \rho_{2} \vec{u}\right)=0
\end{aligned}
$$

E-mail addresses: shyue@ntu.edu.tw (K.-M. Shyue), xiao@es.titech.ac.jp (F. Xiao). 


$$
\begin{aligned}
& \frac{\partial}{\partial t}(\rho \vec{u})+\nabla \cdot(\rho \vec{u} \otimes \vec{u})+\nabla p=0, \\
& \frac{\partial E}{\partial t}+\nabla \cdot(E \vec{u}+p \vec{u})=0, \\
& \frac{\partial \alpha_{1}}{\partial t}+\vec{u} \cdot \nabla \alpha_{1}=0,
\end{aligned}
$$

as an example, for the principal motion of the state variables such as the partial densities, momentum, total energy, and volume fraction, respectively (cf. [1]). Here $\rho_{k}$ and $\alpha_{k} \in[0,1]$ denote in turn the $k$ th phasic density and volume fraction for $k=1,2 ; \alpha_{1}+\alpha_{2}=1$. We have $\rho=\alpha_{1} \rho_{1}+\alpha_{2} \rho_{2}$ representing the total density, $\vec{u}$ the vector of particle velocity, and $p$ the mixture pressure.

To close the system, the constitutive law for each of the fluid phases of interest is assumed to satisfy a Mie-Grüneisen equation of state,

$$
p_{k}\left(\rho_{k}, e_{k}\right)=p_{\infty, k}\left(\rho_{k}\right)+\rho_{k} \Gamma_{k}\left(\rho_{k}\right)\left(e_{k}-e_{\infty, k}\left(\rho_{k}\right)\right),
$$

where $e_{k}$ is the phasic specific internal energy, $\Gamma_{k}=\left.\left(1 / \rho_{k}\right)\left(\partial p_{k} / \partial e_{k}\right)\right|_{\rho_{k}}$ the Grüneisen coefficient, and $p_{\infty, k}, e_{\infty, k}$ are the properly chosen states of the pressure and internal energy along some reference curve in order to match the experimental data of the material being examined. For simplicity, each of the expressions $\Gamma_{k}, p_{\infty, k}$, and $e_{\infty, k}$ is taken as a function of the density only, see Section 5 for an example. As usual, $E=\rho e+\rho \vec{u} \cdot \vec{u} / 2$ is the total energy with the total internal energy defined as a volume-fraction average of the form $\rho e=\sum_{k=1}^{2} \alpha_{k} \rho_{k} e_{k}$.

If the isobaric closure is assumed also in this model, where we have $p_{1}=p_{2}=p$ in a region that contains more than one fluid component, from the total internal energy with (2), it is easy to derive the expression for mixture pressure as

$$
p=\left(\rho e-\sum_{k=1}^{2} \alpha_{k} \rho_{k} e_{\infty, k}\left(\rho_{k}\right)+\sum_{k=1}^{2} \alpha_{k} \frac{p_{\infty, k}\left(\rho_{k}\right)}{\Gamma_{k}\left(\rho_{k}\right)}\right) / \sum_{k=1}^{2} \frac{\alpha_{k}}{\Gamma_{k}\left(\rho_{k}\right)} .
$$

With that, it can be shown that this five-equation model is hyperbolic when each physically relevant value of the state variables of the flow are defined in the region of thermodynamic stability, see [1] for the detail.

As reported in the literature, conventional numerical approach originally developed for single phase compressible flows can be used to solve this system. However, particular attention must be paid when computing the volume fraction. It is well known that even high order Eulerian transport scheme on fixed grids cannot completely remove numerical dissipation which then tends to continuously smear out the initial jump in the volume fraction function. It is obviously problematic for immiscible interfaces. Even worse, it will eventually lead to the failure in identifying the moving interface which separate different materials for long term computations. So, we have to keep the interface sharp or at least recognizable throughout the simulation. To this end, numerical techniques, such as front tracking [5,23,45], anti-diffusion [51,47,48], interface compression [42,54], ALE (Arbitrary Lagrangian-Eulerian) and its variant [3,4,18,25,27,46,49], have been proposed.

In this work, our approach to interface-sharpening is a variant of the THINC (Tangent of Hyperbola for INterface Capturing) scheme that was proposed previously as an advection solver for the sharp resolution of a sub-grid discontinuity of volume fraction in the simulation of incompressible two-phase flow (cf. [2,12,60-62]). Shown in these works, the improved THINC method can get numerical accuracy comparable to the VOF schemes that use explicit geometrical reconstruction, such as PLIC (piecewise linear interface calculation) reconstruction, to retrieve the moving interfaces in incompressible multi-phase flows. Without the geometrical reconstruction, a THINC scheme is just a pure advection scheme, and is thus perhaps the simplest interface-capturing scheme in multi-phase flow simulations.

In the present case with the five-equation model for compressible two-phase flow, in regions near the interfaces, the original THINC scheme is used without any change for the reconstruction of volume fractions at each cell edge. Using the particular reconstruction function, THINC scheme effectively recovers the non-oscillatory sharp resolution of the volume fractions at every time step, and thus completely avoids the appearance of negative volume fractions that always accompanies the high-order Eulerian type schemes over fixed computational grids. With that, assuming the retain of constant cell averages of $\rho_{1}, \rho_{2}, \vec{u}$, and $p$ within a cell, a new reconstruction procedure is derived so as to ensure a consistent modeling of the remaining state variables such as $\alpha_{1} \rho_{1}, \alpha_{2} \rho_{2}, \rho \vec{u}$, and $E$ at the cell edges, see [51] for a similar procedure that was employed in an anti-diffusion type interface-sharpening algorithm. In regions away from the interfaces, where the flow is single phase, standard MUSCL (Monotone Upstream-centered Schemes for Conservation Laws) or WENO (Weighted Essentially Non-Oscillatory) reconstruction may be applied for the interpolation of state variables to high order (cf. [20,41,57]); this will be described further in Section 4.

To find approximate solutions of (1) with (2) and (3) numerically, we use a semi-discrete wave propagation method developed by Ketcheson et al. [15,17] for general hyperbolic systems. In this method, the spatial discretization is constructed by computing fluctuations from the solutions of Riemann problems with the proposed interface-sharpened initial data at cell edges. We employ the strong stability-preserving Runge-Kutta scheme [6,7] for the integration in time, yielding a simple and efficient implementation of the algorithm in the framework of the CLAWPACK software [16,22].

It should be mentioned that our THINC-based interface-sharpening approach is based on fixed underlying grids which is different from time-varying grid approaches such as the Lagrangian or ALE $[3,4,18,25,27,49]$, interface tracking $[32,38,45]$, 
and adaptive moving grid method [53] for sharpening interfaces. In addition, our method is simpler than the ones proposed recently by Shukla et al. [42] and So et al. [51] (see also Appendix B) in the sense that there is no need to introduce additional interface-compression and anti-diffusion source terms to the compressible two-phase flow model, respectively, for the purpose of sharpening interfaces numerically (this may be difficult to do for more complex multiphase flow system and with phase changes, see [40,63], for example). Numerical results presented in Section 5 and Appendix A show the feasibility of the method for practical compressible two-phase flow and moving interface problems.

The format of this paper is outlined as follows. In Section 2, we review the basic idea of a semi-discrete finite volume method in wave-propagation form for hyperbolic conservation laws. In Section 3, we describe the THINC scheme for the sharp reconstruction of interfaces based on a given set of discrete volume fractions. The interface-sharpening algorithm for compressible two-phase flow is discussed in Section 4. Results of some sample validation tests as well as application of the method to practical problems are shown in Section 5. In Appendix A, additional advection benchmark tests are considered, and in Appendix B, a brief review of an anti-diffusion based interface sharpening scheme is described.

\section{Semi-discrete wave propagation method}

We begin our discussion by reviewing a semi-discrete finite volume method in wave-propagation form [15,17] that is essential in our interface-sharpening algorithm for solving the present quasi-conservative five-equation model numerically for compressible two-phase flow. For the ease of the latter description, let us restate (1) in the form

$$
\frac{\partial q}{\partial t}+\sum_{j=1}^{N} \frac{\partial f_{j}(q)}{\partial x^{j}}+\sum_{j=1}^{N} B_{j}(q) \frac{\partial q}{\partial x^{j}}=0
$$

where with $\vec{u}=\left(u_{1}, u_{2}, \ldots, u_{N}\right)$ the terms $q, f_{j}$, and $B_{j}$ are defined by

$$
\begin{aligned}
& q=\left(\alpha_{1} \rho_{1}, \alpha_{2} \rho_{2}, \rho u_{1}, \ldots, \rho u_{N}, E, \alpha_{1}\right)^{T}, \\
& f_{j}=\left(\alpha_{1} \rho_{1} u_{j}, \alpha_{2} \rho_{2} u_{j}, \rho u_{1} u_{j}+p \delta_{1 j}, \ldots, \rho u_{N} u_{j}+p \delta_{N j}, E u_{j}+p u_{j}, 0\right)^{T}, \\
& B_{j}=\operatorname{diag}\left(0,0, \ldots, 0, u_{1} \delta_{1 j}, \ldots, u_{N} \delta_{N j}\right),
\end{aligned}
$$

respectively, $j=1,2, \ldots, N$. Here $\delta_{i j}$ is the Kronecker delta, and $N$ denotes the number of spatial dimension.

\subsection{One-dimensional case}

Consider the one-dimensional case $N=1$ with a uniform grid with fixed mesh spacing $\Delta x^{1}$ and the number of cell in total $M^{1}$ that discretize a spatial domain in the $x^{1}$ coordinate. We use a standard finite-volume formulation in which the value $Q_{i}(t)$ approximates the cell average of the solution over the grid cell $C_{i}: x^{1} \in\left[x_{i-1 / 2}^{1}, x_{i+1 / 2}^{1}\right]$ at time $t$,

$$
Q_{i}(t) \approx \frac{1}{\Delta x^{1}} \int_{x_{i-1 / 2}^{1}}^{x_{i+1 / 2}^{1}} q(x, t) d x
$$

Let $Q(t)=\left(Q_{1}, Q_{2}, \ldots, Q_{M^{1}}\right)^{T}(t)$ be the vector of the approximate solution of $(4)$ at time $t$, and $\mathcal{L}^{1}(Q(t))=$ $\left(\mathcal{L}^{1}\left(Q_{1}\right), \mathcal{L}^{1}\left(Q_{2}\right), \ldots, \mathcal{L}^{1}\left(Q_{M^{1}}\right)\right)^{T}(t)$ be the associated spatial-discretization vector. Then the semi-discrete version of the wave-propagation method is a method-of-lines discretization of (4) that can be written as a system of ODEs (Ordinary Differential Equations)

$$
\frac{\partial Q(t)}{\partial t}=\mathcal{L}^{1}(Q(t))
$$

where each component of $\mathcal{L}^{1}(Q(t))$ is defined by

$$
\mathcal{L}^{1}\left(Q_{i}(t)\right)=-\frac{1}{\Delta x^{1}}\left(\mathcal{A}_{1}^{+} \Delta Q_{i-1 / 2}+\mathcal{A}_{1}^{-} \Delta Q_{i+1 / 2}+\mathcal{A}_{1} \Delta Q_{i}\right)
$$

for $i=1,2, \ldots, M^{1}$. Here $\mathcal{A}^{+} \Delta Q_{i-1 / 2}$ and $\mathcal{A}^{-} \Delta Q_{i+1 / 2}$, are the right- and left-moving fluctuations, respectively, that are entering into the grid cell, and $\mathcal{A}_{1} \Delta Q_{i}$ is the total fluctuation within $C_{i}$. To determine these fluctuations, we need to solve Riemann problems.

Considering the fluctuations $\mathcal{A}_{1}^{ \pm} \Delta Q_{i-1 / 2}$ arising from the edge $(i-1 / 2)$ between cells $i-1$ and $i$, for example. This amounts to solving a Cauchy problem that consists of

$$
\frac{\partial q}{\partial t}+\frac{\partial f_{1}(q)}{\partial x^{1}}+B_{1}(q) \frac{\partial q}{\partial x^{1}}=0
$$


as for the equations and

$$
q\left(x^{1}, t_{0}\right)= \begin{cases}q_{i-1 / 2}^{L} & \text { if } x^{1}<x_{i-1 / 2}^{1} \\ q_{i-1 / 2}^{R} & \text { if } x^{1}>x_{i-1 / 2}^{1}\end{cases}
$$

as for the initial condition at a time $t_{0}$. Here $q_{i-1 / 2}^{L}=\lim _{x \rightarrow x_{(i-1 / 2)^{-}}^{1}} \tilde{q}_{i-1}(x)$ and $q_{i-1 / 2}^{R}=\lim _{x \rightarrow x_{(i-1 / 2)^{+}}^{1}} \tilde{q}_{i}(x)$ are the interpolated states obtained by taking limits of the reconstructed piecewise-continuous function $\tilde{q}_{i-1}(x)$ or $\tilde{q}_{i}(x)$ (each of them are determined based on the set of discrete data $\left\{Q_{i}\left(t_{0}\right)\right\}$, see Sections 3 and 4$)$ to the left and right of the cell edge at $x_{i-1 / 2}^{1}$, respectively.

If an approximate solver is used for the numerical resolution of the above Riemann problem (cf. [1,55]), this would result in three propagating discontinuities that are moving with speeds $s^{1, k}$ and the jumps $\mathcal{W}^{1, k}$ across each of them for $k=1,2,3$, yielding the expression for the fluctuations as

$$
\mathcal{A}_{1}^{ \pm} \Delta Q_{i-1 / 2}=\sum_{k=1}^{3}\left[s^{1, k}\left(q_{i-1 / 2}^{L}, q_{i-1 / 2}^{R}\right)\right]^{ \pm} \mathcal{W}^{1, k}\left(q_{i-1 / 2}^{L}, q_{i-1 / 2}^{R}\right) .
$$

In a similar manner, we may define the fluctuation $\mathcal{A}_{1} \Delta Q_{i}$ based on the Riemann problem with the initial data $q_{i-1 / 2}^{R}$ and $q_{i+1 / 2}^{L}$ at the cell center which gives

$$
\mathcal{A}_{1} \Delta Q_{i}=\sum_{k=1}^{3}\left[s^{1, k}\left(q_{i-1 / 2}^{R}, q_{i+1 / 2}^{L}\right)\right]^{ \pm} \mathcal{W}^{1, k}\left(q_{i-1 / 2}^{R}, q_{i+1 / 2}^{L}\right) ;
$$

this completes the definition of the fluctuations and also the spatial discretization operator $\mathcal{L}^{1}\left(Q_{i}(t)\right)$ in $(5 \mathrm{~b})$. As usual, the notations for the quantities $s^{ \pm}$are set by $s^{+}=\max (s, 0)$ and $s^{-}=\min (s, 0)$.

To integrate the system of ODEs (5a) in time, we employ the SSP (Strong Stability-Preserving) multistage Runge-Kutta scheme [6,7]. That is, in the first-order case we use the Euler forward time discretization as

$$
Q^{n+1}=Q^{n}+\Delta t \mathcal{L}^{1}\left(Q^{n}\right),
$$

where we start with the cell average $Q^{n} \approx Q\left(t_{n}\right)$ at time $t_{n}$, yielding the solution at the next time step $Q^{n+1}$ over $\Delta t=$ $t_{n+1}-t_{n}$. In the second-order case, however, we use the classical two-stage Heun method (or called the modified Euler method) as

$$
\begin{aligned}
& Q^{*}=Q^{n}+\Delta t \mathcal{L}^{1}\left(Q^{n}\right), \\
& Q^{n+1}=\frac{1}{2} Q^{n}+\frac{1}{2} Q^{*}+\frac{1}{2} \Delta t \mathcal{L}^{1}\left(Q^{*}\right) .
\end{aligned}
$$

It is common that the three-stage third-order scheme of the form

$$
\begin{aligned}
& Q^{*}=Q^{n}+\Delta t \mathcal{L}^{1}\left(Q^{n}\right), \\
& Q^{* *}=\frac{3}{4} Q^{n}+\frac{1}{4} Q^{*}+\frac{1}{4} \Delta t \mathcal{L}^{1}\left(Q^{*}\right), \\
& Q^{n+1}=\frac{1}{3} Q^{n}+\frac{2}{3} Q^{* *}+\frac{2}{3} \Delta t \mathcal{L}^{1}\left(Q^{* *}\right)
\end{aligned}
$$

is a preferred one to be used in conjunction with the fifth-order WENO scheme that is employed for the reconstruction of $\tilde{q}_{i}\left(x^{1}\right)$ during the spatial discretization (cf. [41]).

\subsection{Multidimensional case}

To extend the one-dimensional method described above to more space dimensions, here we take a simple dimensionby-dimension approach. Namely, assuming a uniform Cartesian grid with fixed mesh spacing $\Delta x^{1}$ and $\Delta x^{2}$, in the $x^{1}$-, and $x^{2}$-direction, respectively, for example, the semi-discrete wave propagation method in two dimensions can be written of the form

$$
\frac{\partial Q(t)}{\partial t}=\mathcal{L}^{2}(Q(t))
$$

Here both $Q(t)=\left\{Q_{i j}(t)\right\}$ and $\mathcal{L}^{2}(Q(t))=\left\{\mathcal{L}^{2}\left(Q_{i j}(t)\right)\right\}$ are two-dimensional arrays for $i=1,2, \ldots, M^{1}, j=1,2, \ldots, M^{2}$, with $Q_{i j}(t)$ denoting the approximate cell average of the solution for the $(i, j)$ th grid cell over the region $C_{i j}:\left(x^{1}, x^{2}\right) \in$ $\left[x_{i-1 / 2}^{1}, x_{i+1 / 2}^{1}\right] \times\left[x_{j-1 / 2}^{2}, x_{j+1 / 2}^{2}\right]$ at time $t$ as 


$$
Q_{i j}(t) \approx \frac{1}{\Delta x^{1} \Delta x^{2}} \int_{x_{i-1 / 2}^{1}}^{x_{i+1 / 2}^{1}} \int_{x_{j-1 / 2}^{2}}^{x_{j+1 / 2}^{2}} q(x, y, t) d y d x,
$$

and $\mathcal{L}^{2}\left(Q_{i j}(t)\right)$ representing the approximate spatial derivatives of Eqs. (4) for $C_{i j}$ as

$$
\begin{aligned}
\mathcal{L}^{2}\left(Q_{i j}(t)\right)= & -\frac{1}{\Delta x^{1}}\left(\mathcal{A}_{1}^{+} \Delta Q_{i-1 / 2, j}+\mathcal{A}_{1}^{-} \Delta Q_{i+1 / 2, j}+\mathcal{A}_{1} \Delta Q_{i j}\right) \\
& -\frac{1}{\Delta x^{2}}\left(\mathcal{A}_{2}^{+} \Delta Q_{i, j-1 / 2}+\mathcal{A}_{2}^{-} \Delta Q_{i, j+1 / 2}+\mathcal{A}_{2} \Delta Q_{i j}\right) .
\end{aligned}
$$

Note that the fluctuations $\mathcal{A}_{1}^{+} \Delta Q_{i-1 / 2, j}, \mathcal{A}_{1}^{-} \Delta Q_{i+1 / 2, j}$ and $\mathcal{A}_{1} \Delta Q_{i j}$ are obtained by solving the one-dimensional Riemann problems in the direction normal to the $x^{1}$-axis (see (6)), while the fluctuations $\mathcal{A}_{2}^{+} \Delta Q_{i, j-1 / 2}, \mathcal{A}_{2}^{-} \Delta Q_{i, j+1 / 2}$ and $\mathcal{A}_{2} \Delta Q_{i j}$ can be computed in a similar manner by solving the one-dimensional Riemann problems of in the direction normal to the $x^{2}$-axis with

$$
\frac{\partial q}{\partial t}+\frac{\partial f_{2}(q)}{\partial x^{2}}+B_{2}(q) \frac{\partial q}{\partial x^{2}}=0
$$

as for the equations and with chosen reconstructed data as for the initial condition. The SSP Runge-Kutta scheme can be employed also for the integration of the semi-discrete scheme (9) in time.

It should be mentioned that the class of dimension-by-dimension semi-discrete method considered here would only give second order results when it is applied to solve general nonlinear hyperbolic systems, even if the fifth-order WENO reconstruction scheme is used in the spatial discretization, and the third order Runge-Kutta method is employed in time integration, see [64] for the details. We will not discuss the fully multidimensional spatial discretization in wave-propagation form here which is beyond the scope of this paper (see $[20,41]$ and references cited therein for more discussions on how this can be done), but will devote our attention to the devise of a new reconstruction procedure for the purpose of sharpening interfaces from compressible two-phase flows.

\subsection{Include source terms}

To end this section, we comment that if $x^{1}$ is the axisymmetric direction and $u_{1}$ is the radial velocity, an axisymmetric version of the five-equation model (4) in one space dimension can be written as

$$
\frac{\partial q}{\partial t}+\frac{\partial f_{1}(q)}{\partial x^{1}}+B_{1}(q) \frac{\partial q}{\partial x^{1}}=\psi(q)
$$

where $\psi$ is the source term derived directly from the geometric simplification, yielding

$$
\psi=-\frac{\kappa}{x^{1}}\left(\alpha_{1} \rho_{1} u_{1}, \alpha_{2} \rho_{2} u_{1}, \rho u_{1}^{2}, E u_{1}+p u_{1}, 0\right)^{T} .
$$

Here in the case of a 2D radially or 3D spherically symmetric flow, the quantity $\kappa$ takes 1 or 2, respectively.

Now, to find approximate solutions of (10) numerically, in this work, we take a simple unsplit approach (cf. [20]) in that the semi-discrete scheme (5) is employed again, but the term $\mathcal{L}^{1}(Q(t))$ on the right-hand side of the equation is modified to include the effect of source term $\psi$ as

$$
\mathcal{L}^{1}\left(Q_{i}(t)\right):=\mathcal{L}^{1}\left(Q_{i}(t)\right)+\psi\left(Q_{i}(t)\right)
$$

We then continue by employing an SSP Runge-Kutta method as usual to integrate the resulting ODEs in time for the updated solution at the next time step, see Section 5.1 for a sample result obtained using the method.

\section{THINC reconstruction scheme}

The reconstruction for any physical field to identify a sub-grid discontinuity can be derived using the THINC formulation for volume fraction function as a building block. To describe the basic idea, we start with the one-dimensional case, and for simplicity rather than using $x^{1}$ for the spatial variable and $\alpha_{1}\left(x^{1}, t\right)$ for the volume fraction as in Section 2, we take the often-employed symbols $x$ and $\phi(x, t)$ for that matter instead. Then as before we define the approximate value of the cell-average of $\phi(x, t)$ over the grid cell $C_{i}$ at time $t$ by

$$
\bar{\phi}_{i}(t) \approx \frac{1}{\Delta x_{i}} \int_{x_{i-1 / 2}}^{x_{i+1 / 2}} \phi(x, t) d x,
$$


where $\Delta x_{i}=x_{i+1 / 2}-x_{i-1 / 2}$ is the mesh size of the cell; assuming a non-uniform discretization of the spatial domain. Recall that being a volume fraction of a specified fluid, say fluid $\mathcal{F}$, we have the values of $\bar{\phi}_{i}(t)$ as follows,

$$
\bar{\phi}_{i}(t)= \begin{cases}1 & \text { if } C_{i} \text { is filled with fluid } \mathcal{F}, \\ \alpha \in(0,1) & \text { if an interface lies in } C_{i}, \\ 0 & \text { if there is no fluid } \mathcal{F} \text { in } C_{i} .\end{cases}
$$

In practice, we identify an "interface cell" where the volume fraction satisfies,

(i) $\epsilon<\bar{\phi}_{i}(t)<1-\epsilon$,

(ii) $\left(\bar{\phi}_{i+1}(t)-\bar{\phi}_{i}(t)\right)\left(\bar{\phi}_{i}(t)-\bar{\phi}_{i-1}(t)\right)>0$,

where $\epsilon$ is a small positive parameter (e.g., $10^{-4}$ ). Note that the latter condition (ii) is a monotonicity constraint on the data near the interfaces; this is introduced as a measure to prevent from incurring spurious oscillations in the method.

Now given the volume fraction for the interface cell $C_{i}$, we seek a reconstruction that mimics the sub-grid structure of the jump between 0 and 1 in the volume fraction function. For a fixed time $t$ and $x \in\left[x_{i-1 / 2}, x_{i+1 / 2}\right]$, the following hyperbolic tangent function is well suited for this purpose,

$$
\Phi_{i}(x)=\frac{1}{2}\left[1+\sigma_{i} \tanh \left(\beta\left(\frac{x-x_{i-1 / 2}}{\Delta x_{i}}-\tilde{x}_{i}\right)\right)\right],
$$

where $\sigma_{i}=\operatorname{sgn}\left(\Delta \bar{\phi}_{i}(t)\right)$ is the sign function of the variable $\Delta \bar{\phi}_{i}(t)=\bar{\phi}_{i+1}(t)-\bar{\phi}_{i-1}(t)$, and $\beta$ is a prescribed parameter to control the slope and thickness of the jump which can be determined flexibly in practice (here we take $\beta=2.3$ in all the tests considered in Section 5).

Note that the only unknown in (14a) is the center $\tilde{x}_{i}$. If we assume the conservation of volume fraction of the form

$$
\frac{1}{\Delta x_{i}} \int_{x_{i-1 / 2}}^{x_{i+1 / 2}} \Phi_{i}(x) d x=\bar{\phi}_{i}(t)
$$

it can be determined uniquely as

$$
\tilde{x}_{i}=\frac{1}{2 \beta} \ln \left[\frac{\exp \left(\beta\left(1+\sigma_{i}-2 \bar{\phi}_{i}\right) / \sigma_{i}\right)}{1-\exp \left(\beta\left(1-\sigma_{i}-2 \bar{\phi}_{i}\right) / \sigma_{i}\right)}\right] .
$$

It should be mentioned that using (14) as a building block, we may reconstruct any physical field $\psi(x, t)$ for $x \in$ $\left[x_{i-1 / 2}, x_{i+1 / 2}\right]$ where it has a jump $\Delta \psi_{i}=\psi_{\max }-\psi_{\min }$ by,

$$
\Psi_{i}(x)=\psi_{\min }+\Phi_{i}(x) \Delta \psi_{i} .
$$

Here lower and upper bounds of the jump $\psi_{\min }$ and $\psi_{\max }$ can be determined from the neighboring cells such as $\psi_{\min }=$ $\min \left(\psi_{i-1 / 2}^{L}, \psi_{i+1 / 2}^{R}\right)$ and $\psi_{\max }=\max \left(\psi_{i-1 / 2}^{L}, \psi_{i+1 / 2}^{R}\right)$, where $\psi_{i-1 / 2}^{L}$ denotes the value computed from the reconstruction over the left-side cell $\left[x_{i-3 / 2}, x_{i-1 / 2}\right]$ and $\psi_{i+1 / 2}^{R}$ from the right-side cell $\left[x_{i+1 / 2}, x_{i+3 / 2}\right]$.

Reconstruction (15) can be applied to various physical variables, such as primitive variables, characteristic variables and flux functions. As long as the THINC reconstruction (15) is built up, we can get directly the values at the cell boundaries as a function of time $t$ through either a point mapping or a trajectory integration for a wave propagation (transport) equation. In the latter instance, we consider the following wave equation as an example,

$$
\frac{\partial \psi}{\partial t}+u \frac{\partial \psi}{\partial x}=0
$$

where $u$ is the characteristic velocity field. We split $u$ into right-moving and left-moving parts, i.e., $u=u^{+}+u^{-}$where $u^{+}=\max (u, 0)$ and $u^{-}=\min (u, 0)$. The solution at the right-side of cell boundary $x_{i-1 / 2}$ reads

$$
\begin{aligned}
\psi_{i-1 / 2}^{R}(t) & =\Psi_{i}\left(x_{i-1 / 2}-u_{i-1 / 2}^{-} t\right) \\
& =\psi_{\min }+\Phi_{i}\left(x_{i-1 / 2}-u_{i-1 / 2}^{-} t\right) \Delta \psi_{i} \\
& =\psi_{\min }+\frac{1}{2}\left[1+\sigma_{i} \tanh \left(\beta\left(\frac{u_{i-1 / 2}^{-} t}{\Delta x_{i}}-\tilde{x}_{i}\right)\right)\right] \Delta \psi_{i},
\end{aligned}
$$

while that at the left-side of cell boundary $x_{i+1 / 2}$ reads 


$$
\begin{aligned}
\psi_{i+1 / 2}^{L}(t) & =\Psi_{i}\left(x_{i+1 / 2}-u_{i+1 / 2}^{+} t\right) \\
& =\psi_{\min }+\Phi_{i}\left(x_{i+1 / 2}-u_{i+1 / 2}^{+} t\right) \Delta \psi_{i} \\
& =\psi_{\min }+\frac{1}{2}\left[1+\sigma_{i} \tanh \left(\beta\left(1-\frac{u_{i+1 / 2}^{+} t}{\Delta x_{i}}-\tilde{x}_{i}\right)\right)\right] \Delta \psi_{i},
\end{aligned}
$$

which can be used to any approximate Riemann solver in a full- or semi-discrete form, see the formulas given in [2] for that purpose also.

For the mass conservation law,

$$
\frac{\partial \psi}{\partial t}+\frac{\partial f}{\partial x}=0,
$$

where $f=u \psi$ is the flux function, we have the total leftward flux across cell boundary $x_{i-1 / 2}$ over $t$ as,

$$
\begin{aligned}
\hat{f}_{i-1 / 2}^{-}(t) & =\int_{0}^{t} f\left(x_{i-1 / 2}, t\right) d t=\int_{x_{i-1 / 2}-u_{i-1 / 2}^{-} t}^{x_{i-1 / 2}} \Psi_{i}(x) d x \\
& =\psi_{0} u_{i-1 / 2}^{-} t-\frac{\sigma_{i} \Delta x_{i}}{2 \beta} \ln \left[\frac{\cosh \left(\beta\left(\tilde{x}_{i}+\frac{u_{i-1 / 2}^{-} t}{\Delta x_{i}}\right)\right)}{\cosh \left(\beta \tilde{x}_{i}\right)}\right] \Delta \psi_{i} .
\end{aligned}
$$

Analogously, the rightward flux across $x_{i+1 / 2}$ is

$$
\begin{aligned}
\hat{f}_{i+1 / 2}^{+}(t) & =\int_{0}^{t} f\left(x_{i+1 / 2}, t\right) d t=\int_{x_{i+1 / 2}-u_{i+1 / 2}^{+} t}^{x_{i+1 / 2}} \Psi_{i}(x) d x \\
& =\psi_{0} u_{i+1 / 2}^{+} t-\frac{\sigma_{i} \Delta x_{i}}{2 \beta} \ln \left[\frac{\cosh \left(\beta\left(1-\tilde{x}_{i}-\frac{u_{i+1 / 2}^{+} t}{\Delta x_{i}}\right)\right)}{\cosh \left(\beta\left(1-\tilde{x}_{i}\right)\right)}\right] \Delta \psi_{i} .
\end{aligned}
$$

where $\psi_{0}=\left(\psi_{\min }+\psi_{\max }\right) / 2$. So, a one-step finite volume formulation to transport mass can be written as,

$$
\bar{\psi}_{i}(t)=\bar{\psi}_{i}(0)-\frac{1}{\Delta x_{i}}\left(\hat{f}_{i+1 / 2}(t)-\hat{f}_{i-1 / 2}(t)\right),
$$

which is used in the existing THNIC method for incompressible flows.

To extend the above THINC reconstruction scheme to more than one space dimension, here we take a simple dimensionby-dimension approach as proposed in Xiao et al. [60]; this one-dimensional version of the method works quite well with the semi-discrete wave propagation method described in Section 2 and also the interface-sharpening reconstruction scheme for compressible two-phase flow described next.

\section{Interface sharpening algorithm}

In each time step, our interface-sharpening algorithm for compressible two-phase flow problems consists of the following steps:

(1) Reconstruct a piecewise polynomial function, denoted by $\tilde{q}\left(x^{i}, t_{n}\right)$, for all $x^{i}$ based on standard MUSCL/WENO reconstruction procedure from the cell average $Q^{n}$ at time $t_{n}$ to more than first order.

(2) Modify $\tilde{q}\left(x^{i}, t_{n}\right)$ for interface cells using a variant of THINC scheme from $Q^{n}$ to sharpen the resolution of interfaces.

(3) Solve Riemann problems with interpolated initial data from $\tilde{q}\left(x^{i}, t_{n}\right)$ obtained in steps 1 and 2 for spatial discretization.

(4) Employ a semi-discrete method in wave propagation form to update $Q^{n}$ from the current time to the next $Q^{n+1}$ over a time step $\Delta t$.

Note that if step 2 is omitted in the above algorithm, it is simply the standard semi-discrete method proposed by Ketcheson et al. [15,17] for general hyperbolic systems with new applications to compressible two-phase flow, see Section 2. In this instance, the implementation of the MUSCL/WENO reconstruction scheme as suggested in $[13,20,34]$ for step 1 is enough for that matter. Our goal here is to describe step 2 of the reconstruction procedure that is essential to our interface-sharpening algorithm. 


\subsection{Homogeneous-equilibrium-consistent reconstruction scheme}

We begin step 2 by employing the one-dimensional THINC scheme described in Section 3 for the reconstruction of the volume fraction function $q^{(N+4)}=\alpha_{1}$, in the five-equation model (4). Suppose that with $Q^{n}$ given at a time $t_{n}$, we have obtained the interpolated volume fraction for the $i$ th cell at the edges $\left(\alpha_{1}\right)_{i-1 / 2}^{R}$ and $\left(\alpha_{1}\right)_{i+1 / 2}^{L}$ based on the relations $(16 a)$ and (16b) at $t=0$, respectively.

With that, to construct the sub-grid structure of the partial density, we follow the approach proposed by So et al. [51] (see also Appendix B and [54]) in that the phasic densities $\rho_{1}$ and $\rho_{2}$ are assumed to remain constants within the cells during the reconstruction step, yielding the definition of the cell-edge states for the partial densities, i.e., the first and second component of $q ; q^{(k)}=\alpha_{k} \rho_{k}$ for $k=1,2$, as

$$
\begin{aligned}
& \left(\alpha_{k} \rho_{k}\right)_{i-1 / 2}^{R}=\left(\alpha_{k} \rho_{k}\right)_{i}+\left(\rho_{k}\right)_{i}\left[\left(\alpha_{k}\right)_{i-1 / 2}^{R}-\left(\alpha_{k}\right)_{i}\right], \\
& \left(\alpha_{k} \rho_{k}\right)_{i+1 / 2}^{L}=\left(\alpha_{k} \rho_{k}\right)_{i}+\left(\rho_{k}\right)_{i}\left[\left(\alpha_{k}\right)_{i+1 / 2}^{L}-\left(\alpha_{k}\right)_{i}\right] .
\end{aligned}
$$

Here $\left(\alpha_{k}\right)_{i}\left(\rho_{k}\right)_{i}$, and $\left(\alpha_{k} \rho_{k}\right)_{i}$ are the cell-average variables obtained from $Q_{i}^{n}$ in an interface cell $C_{i}$.

Now to find the reconstructed states for the total momentum $q^{(j+2)}=\rho u_{j}$ for $j=1,2, \ldots, N$, the velocity field $\vec{u}=$ $\left(u_{1}, u_{2}, \ldots, u_{N}\right)$ is assumed to remain unchanged within the cells also; this is true in a region for the contact discontinuity (the case we are interested in this work). Thus, we have

$$
\begin{aligned}
& (\rho \vec{u})_{i-1 / 2}^{R}=(\rho \vec{u})_{i}+\vec{u}_{i}\left(\rho_{i-1 / 2}^{R}-\rho_{i}\right), \\
& (\rho \vec{u})_{i+1 / 2}^{L}=(\rho \vec{u})_{i}+\vec{u}_{i}\left(\rho_{i+1 / 2}^{L}-\rho_{i}\right),
\end{aligned}
$$

where $\rho_{i-1 / 2}^{R}=\sum_{k=1}^{2}\left(\alpha_{k} \rho_{k}\right)_{i-1 / 2}^{R}$ and $\rho_{i+1 / 2}^{L}=\sum_{k=1}^{2}\left(\alpha_{k} \rho_{k}\right)_{i+1 / 2}^{L}$ denotes the reconstructed mixture density at the left- and right-edge of the grid cell $i$, respectively.

Finally, to reconstruct the total energy $q^{(N+3)}=E$, we assume further the pressure equilibrium within interface cells, yielding

$$
\begin{aligned}
& E_{i-1 / 2}^{R}=E_{i}+K_{i}\left(\rho_{i-1 / 2}^{R}-\rho_{i}\right)+\sum_{k=1}^{2}\left(\rho_{k} e_{k}\right)_{i}\left[\left(\alpha_{k}\right)_{i-1 / 2}^{R}-\left(\alpha_{k}\right)_{i}\right], \\
& E_{i+1 / 2}^{L}=E_{i}+K_{i}\left(\rho_{i+1 / 2}^{L}-\rho_{i}\right)+\sum_{k=1}^{2}\left(\rho_{k} e_{k}\right)_{i}\left[\left(\alpha_{k}\right)_{i+1 / 2}^{L}-\left(\alpha_{k}\right)_{i}\right],
\end{aligned}
$$

where $K_{i}=\vec{u}_{i} \cdot \vec{u}_{i} / 2$ denotes the specific kinetic energy in cell $C_{i}$. Note that since the above sub-grid reconstruction procedure (18) makes use of the basic homogeneous-equilibrium assumptions of the underlying continuum model (1), it should be pertinent to call it a homogeneous-equilibrium-consistent reconstruction scheme.

\subsection{Interface only problem}

To see how our algorithm works for sharpening interfaces, it is instructive to consider an interface only problem where the initial data consists of uniform pressure $p=p_{0}$, constant velocity $\vec{u}=\vec{u}_{0}$, and constant phasic densities $\rho_{k}=\rho_{k 0}$ for $k=1,2$ throughout the domain, while there are jumps on the other variables such as partial densities and volume fractions across some interfaces. Without loss of generality, we consider a one-dimensional problem with a positive velocity $u_{1}=$ $u_{0}>0$. In this case, suppose that a 3-wave HLLC approximate solver (cf. $[9,44,55]$ ) is used in step 3 of the algorithm for solving the Riemann problem (6) (similar result follows if a Roe solver [1] is employed instead), it is easy to find the fluctuations for each cell $C_{i}, i=1,2, \ldots, M^{1}$, as

$$
\begin{aligned}
& \mathcal{A}_{1}^{-} \Delta Q_{i+1 / 2}=0, \\
& \mathcal{A}_{1}^{+} \Delta Q_{i-1 / 2}=u_{0}\left(q_{i-1 / 2}^{R}-q_{i-1 / 2}^{L}\right), \\
& \mathcal{A}_{1} \Delta Q_{i}=u_{0}\left(q_{i+1 / 2}^{L}-q_{i-1 / 2}^{R}\right) .
\end{aligned}
$$

Inserting the above expression for fluctuations into (5b), we have the spatial discretization of this interface only problem:

$$
\mathcal{L}^{1}\left(Q_{i}(t)\right)=-\frac{1}{\Delta x^{1}} u_{0}\left(q_{i+1 / 2}^{L}-q_{i-1 / 2}^{L}\right) .
$$

Now if the Euler method (8a) is employed for the time integration in (5a), together with the above spatial discretization term the cell average $Q_{i}^{n}$ is updated by

$$
Q_{i}^{n+1}=Q_{i}^{n}-\frac{\Delta t}{\Delta x^{1}} u_{0}\left(q_{i+1 / 2}^{L}-q_{i-1 / 2}^{L}\right),
$$


or equivalently by

$$
\left[\begin{array}{c}
\alpha_{1} \rho_{1} \\
\alpha_{2} \rho_{2} \\
\rho u_{1} \\
E \\
\alpha_{1}
\end{array}\right]_{i}^{n+1}=\left[\begin{array}{c}
\alpha_{1} \rho_{1} \\
\alpha_{2} \rho_{2} \\
\rho u_{1} \\
E \\
\alpha_{1}
\end{array}\right]_{i}^{n}-\frac{\Delta t}{\Delta x^{1}} u_{0}\left[\begin{array}{c}
\left(\alpha_{1} \rho_{1}\right)_{i+1 / 2}^{L}-\left(\alpha_{1} \rho_{1}\right)_{i-1 / 2}^{L} \\
\left(\alpha_{2} \rho_{2}\right)_{i+1 / 2}^{L}-\left(\alpha_{2} \rho_{2}\right)_{i-1 / 2}^{L} \\
u_{0}\left(\rho_{i+1 / 2}^{L}-\rho_{i-1 / 2}^{L}\right) \\
E_{i+1 / 2}^{L}-E_{i-1 / 2}^{L} \\
\left(\alpha_{1}\right)_{i+1 / 2}^{L}-\left(\alpha_{1}\right)_{i-1 / 2}^{L}
\end{array}\right]
$$

Here we have assumed a consistent approximation of the true solution of the problem from the earlier time steps so that the equilibrium conditions $p^{m}=p_{0}, u_{1}^{m}=u_{0}$, and $\rho_{k}^{m}=\rho_{k 0}, k=1,2$, are fulfilled for $0 \leqslant m \leqslant n$.

With that, if we substitute the first two components of (19) into the third one, we arrive at readily the expected state of the particle velocity

$$
\left(u_{1}\right)_{i}^{n+1}=\left(u_{1}\right)_{i}^{n}=u_{0} .
$$

When we continue applying this result to the fourth component of (19), after simple algebraic manipulations, we get the update of the total internal energy

$$
\sum_{k=1}^{2}\left(\alpha_{k} \rho_{k} e_{k}\right)_{i}^{n+1}=\sum_{k=1}^{2}\left(\alpha_{k} \rho_{k} e_{k}\right)_{i}^{n}-\frac{\Delta t}{\Delta x^{1}} u_{0}\left[\left(\alpha_{k} \rho_{k} e_{k}\right)_{i+1 / 2}^{L}-\left(\alpha_{k} \rho_{k} e_{k}\right)_{i-1 / 2}^{L}\right],
$$

or alternatively

$$
\begin{aligned}
\left(\alpha_{k}\left(\frac{p-p_{\infty, k}}{\Gamma_{k}}+\rho_{k} e_{\infty, k}\right)\right)_{i}^{n+1}= & \left(\alpha_{k}\left(\frac{p-p_{\infty, k}}{\Gamma_{k}}+\rho_{k} e_{\infty, k}\right)\right)_{i}^{n}-\frac{\Delta t}{\Delta x^{1}} u_{0} \\
& \cdot\left[\left(\alpha_{k}\left(\frac{p-p_{\infty, k}}{\Gamma_{k}}+\rho_{k} e_{\infty, k}\right)\right)_{i+1 / 2}^{L}-\left(\alpha_{k}\left(\frac{p-p_{\infty, k}}{\Gamma_{k}}+\rho_{k} e_{\infty, k}\right)\right)_{i-1 / 2}^{L}\right],
\end{aligned}
$$

when it is written componentwise for $k=1,2$ and with the Mie-Grüneisen equation of state (2). Note that based on the results for $\alpha_{k} \rho_{k}$ and $\alpha_{1}$ from (19), we find the phasic density $\rho_{k}^{n+1}$ retains its expected value $\rho_{k}^{n}$ for $k=1,2$. Using this fact together with $p^{n}=p_{0}$ and the consistent reconstruction of $\rho_{k} e_{k}$ at each cell edges, it is not difficult to show the fulfillment of pressure equilibrium $p^{n+1}=p_{0}$ from the above equation for internal energy.

Having these results in mind, it should be easy to comprehend that the numerical resolution of volume fractions gives the basic characterization of the discontinuous interfacial profile for both density and total internal energy in which the THINC reconstruction scheme proposed here may be advantageous for some practical problems with immiscible interfaces; this will be justified numerically in Section 5 .

\section{Numerical results}

We now present sample numerical results obtained using our semi-discrete wave propagation method with and without THINC-based solution reconstruction described in the previous sections for compressible two-phase flows in one and two space dimensions. In all the cases considered here, we have used a second-order MUSCL method with the HLLC approximate Riemann solver in step 1, a second-order SSP method in step 4, and the Courant number $v=1 / 2$ (cf. [20]), while performing the runs. To demonstrate more proof of competitiveness of the approach, we have also included results obtained using the anti-diffusion interface sharpening technique (see Appendix B for a brief overview of the method).

\subsection{One-dimensional case}

Example 5.1. We begin by considering a simple interface only problem in one dimension where the exact solution of the problem consists of a square liquid column evolving in gas with uniform equilibrium pressure $p=p_{0}=10^{5} \mathrm{~Pa}$ and constant particle velocity $u_{1}=u_{0}=100 \mathrm{~m} / \mathrm{s}$ in a shock tube of one meter. In this test, inside the region $x^{1} \in[0.4,0.6] \mathrm{m}$ the fluid is nearly liquid that contains a uniformly distributed small amount of the gas volume fraction $\alpha_{2}=10^{-8}$, and it is nearly gas that contains the liquid volume fraction $\alpha_{1}=10^{-8}$ elsewhere; the density for the pure liquid and gas phase in the domain are $\rho_{1}=1000 \mathrm{~kg} / \mathrm{m}^{3}$ and $\rho_{2}=1 \mathrm{~kg} / \mathrm{m}^{3}$, respectively. We use the stiffened gas equation of state to model the thermodynamic behavior of liquid and gas where the material-dependent functions appeared in (2) are $\Gamma_{k}=\gamma_{k}-1$, $p_{\infty, k}=-\gamma_{k} \mathcal{B}_{k}$, and $e_{\infty, k}=0$ with the parameter values taken in turn to be $\gamma_{1}=4.4, \mathcal{B}_{1}=6 \times 10^{8} \mathrm{~Pa}$, and $\gamma_{2}=1.4, \mathcal{B}_{2}=0$ for the liquid and gas phases.

We carried out the computation using the proposed semi-discrete method with and without THINC reconstruction. Periodic boundary condition is used on the left and right boundaries during the computations. Fig. 1 shows numerical results at 

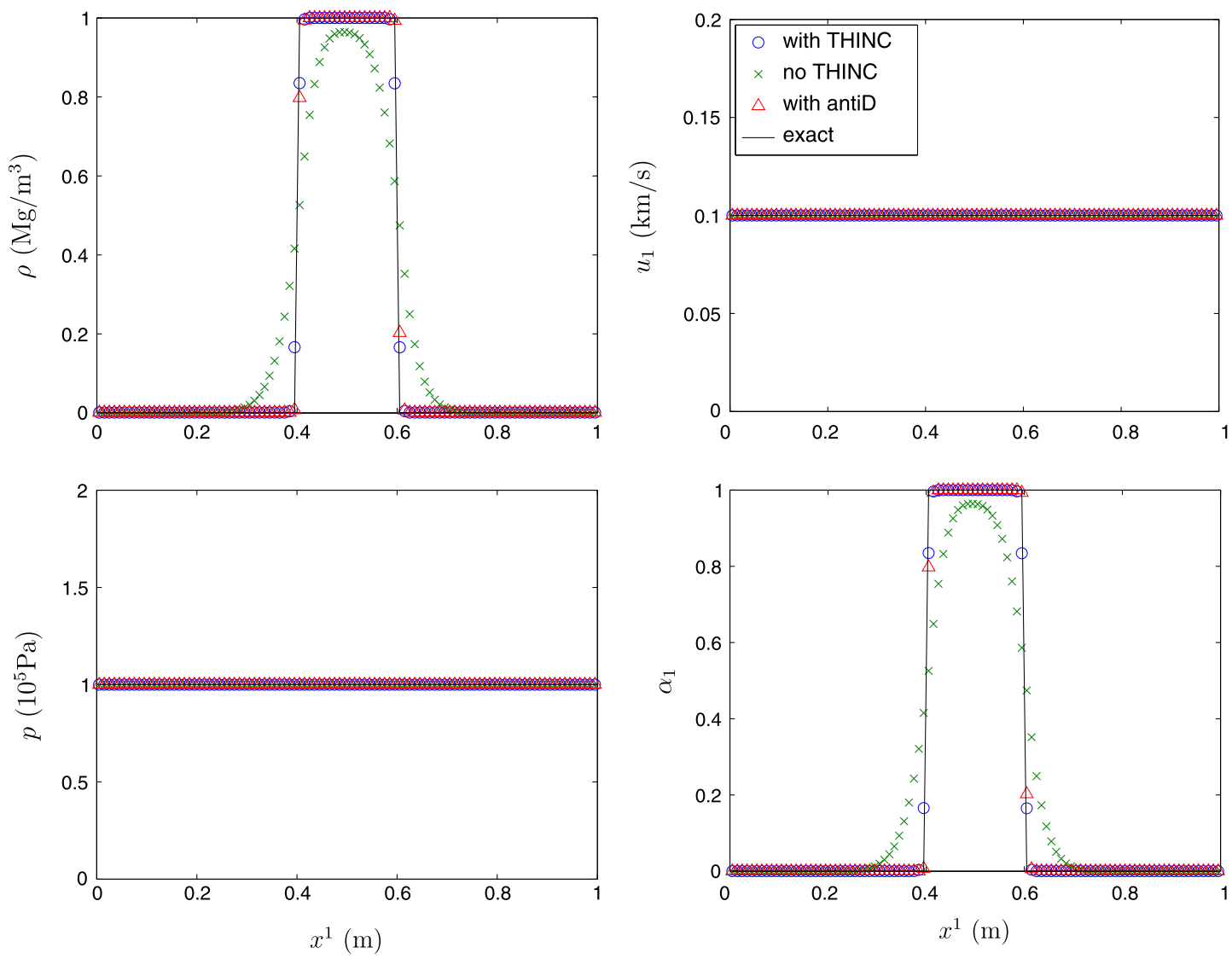

Fig. 1. Numerical results for a passive advection of a square liquid column at time $t=10 \mathrm{~ms}$. The solid line is the exact solution and the points show the computed solution with 100 mesh points obtained using each of the methods with and without THINC reconstruction; in each graph the anti-diffusion results (marked by triangles) are included also as for comparison.

time $t=10 \mathrm{~ms}$ using a 100 mesh; for comparison purposes results obtained using a wave-propagation based anti-diffusion method are included also. From the figure, it is easy to see that, with each of the methods, we have retained the correct pressure equilibrium and particle velocity, without introducing any spurious oscillations near the interfaces. Comparing with the exact solution, we observe an improved resolution of the interfaces for the density and volume fraction when the interface-sharpening method (with either the THINC- or anti-diffusion-based) is employed in the simulation.

Example 5.2. Our next example is a two-phase impact problem proposed by Saurel and Abgrall [39] (see also [43]). Initially, under the atmospheric condition (i.e., with uniform pressure $p_{0}=10^{5} \mathrm{~Pa}$ and temperature $T_{0}=300 \mathrm{~K}$ throughout the domain), there is a rightward going copper plate with the speed $u_{1}=1500 \mathrm{~m} / \mathrm{s}$ interacting with a solid explosive at rest on the right of the plate, see Fig. 2 for illustration. In this problem, as in the previous test, there is a uniformly distributed small amount of volume fraction $\alpha_{2}=10^{-8}$ and $\alpha_{1}=10^{-8}$ inside the copper and explosive, respectively. To model the material properties of the copper and (solid) explosive, we use the Cochran-Chan equation of state that in (2) we set the same $\Gamma_{k}$ as in the stiffened gas case, but with $p_{\infty, k}, e_{\infty, k}$ defined by

$$
\begin{aligned}
& p_{\infty, k}\left(\rho_{k}\right)=\mathcal{B}_{1 k}\left(\frac{\rho_{0 k}}{\rho_{k}}\right)^{-\mathcal{E}_{1 k}}-\mathcal{B}_{2 k}\left(\frac{\rho_{0 k}}{\rho_{k}}\right)^{-\mathcal{E}_{2 k}}, \\
& e_{\infty, k}\left(\rho_{k}\right)=\frac{-\mathcal{B}_{1 k}}{\rho_{0 k}\left(1-\mathcal{E}_{1 k}\right)}\left[\left(\frac{\rho_{0 k}}{\rho_{k}}\right)^{1-\mathcal{E}_{1 k}}-1\right]+\frac{\mathcal{B}_{2 k}}{\rho_{0 k}\left(1-\mathcal{E}_{2 k}\right)}\left[\left(\frac{\rho_{0 k}}{\rho_{k}}\right)^{1-\mathcal{E}_{2 k}}-1\right]-C_{v k} T_{0} .
\end{aligned}
$$

Here $\gamma_{k}, \mathcal{B}_{1 k}, \mathcal{B}_{2 k}, \mathcal{E}_{1 k}, \mathcal{E}_{2 k}, C_{v k}$, and $\rho_{0 k}$ are material-dependent quantities, see Table 1 for a typical set of numerical values for copper and explosive considered here (cf. [26,29,59]).

It is known that the exact solution of this impact problem is composed of a leftward-going shock wave to the copper, a rightward-going shock waves to the inert explosive, and a material interface lying in between that separates these two different materials (cf. [39]). We run this problem using the same numerical methods as performed in the previous examples for the single interface only problem but with a 200 grid. Fig. 2 shows the resulting solutions at time $t=85 \mu$ s for the 
Table 1

Material quantities for solid copper $(k=1)$ and explosive $(k=2)$ in the Cochran-Chan equation of state (20).

\begin{tabular}{clccccc}
\hline$k$ & $\rho_{0 k}\left(\mathrm{~kg} / \mathrm{m}^{3}\right)$ & $\mathcal{B}_{1 k}(\mathrm{GPa})$ & $\mathcal{B}_{2 k}(\mathrm{GPa})$ & $\mathcal{E}_{1 k}$ & $\mathcal{E}_{2 k}$ & $\boldsymbol{C}_{v k}(\mathrm{~J} / \mathrm{kg} \mathrm{K})$ \\
\hline 1 & 8900 & 145.67 & 147.75 & 2.99 & 3.99 & 393 \\
2 & 1840 & 12.87 & 13.42 & 4.1 & 3.1 & 1.93 \\
\hline
\end{tabular}
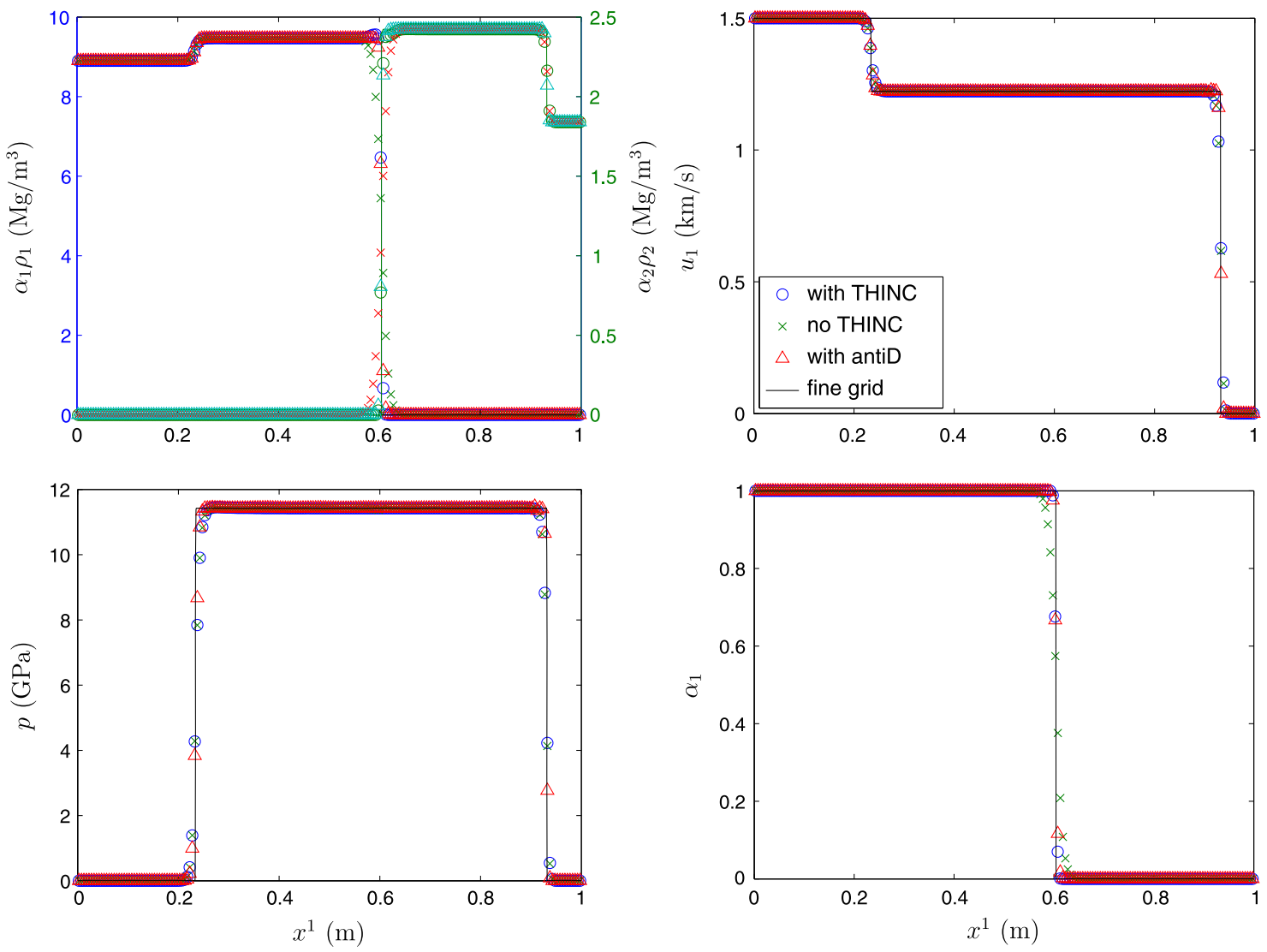

Fig. 2. Numerical results for a two-phase (solid explosive-copper) impact problem at time $t=85 \mu$ s. The solid line is the fine grid solution computed by 5000 meshes, and the points show the solutions with 200 meshes.

density, velocity, pressure, and the copper volume fraction. By comparing the computed solutions with the fine grid one obtained using the THINC-based interface-sharpening method but with 5000 meshes, we see good agreement of the solution behaviors in all the methods under concerned with the correct shock speeds and free of spurious oscillations in the pressure near the interface. With the THINC reconstruction, we again observe some improvement on the structure of the density and volume fraction near the interface. Notice that there is a slight overshoot on the phasic density $\alpha_{1} \rho_{1}$ on the left of the interface; this error diminishes however when the mesh is refined. This overshoot does not appear in the present anti-diffusion results.

Example 5.3. We continue our test by considering the interaction of a shock wave in molybdenum with an encapsulated MORB (Mid-Ocean Ridge Basalt) liquid. As in [30,31,43], we use (2) to model the thermodynamic behavior of MORB and molybdenum with $\Gamma_{k}, p_{\infty, k}$, and $e_{\infty, k}$ defined as follows:

$$
\begin{aligned}
& \Gamma_{k}\left(\rho_{k}\right)=\Gamma_{0 k}\left(\frac{\rho_{0 k}}{\rho_{k}}\right)^{\eta_{k}}, \\
& p_{\infty, k}\left(\rho_{k}\right)=p_{0}+\frac{\rho_{0 k} c_{0 k}^{2}\left(1-\frac{\rho_{0 k}}{\rho_{k}}\right)}{\left[1-\zeta_{k}\left(1-\frac{\rho_{0 k}}{\rho_{k}}\right)\right]^{2}}, \\
& e_{\infty, k}\left(\rho_{k}\right)=e_{0}+\frac{1}{2 \rho_{0 k}}\left(1-\frac{\rho_{0 k}}{\rho_{k}}\right)\left(p_{0}+p_{\infty, k}\left(\rho_{k}\right)\right) .
\end{aligned}
$$


Table 2

Material quantities for MORB $(k=1)$ and molybdenum $(k=2)$ in the Mie-Grüneisen equation of state (2) with $(21)$.

\begin{tabular}{llllll}
\hline$k$ & $\rho_{0 k}\left(\mathrm{~kg} / \mathrm{m}^{3}\right)$ & $c_{0 k}(\mathrm{~m} / \mathrm{s})$ & $\zeta_{k}$ & $\gamma_{k}$ & \\
\hline 1 & 2660 & 2100 & 1.68 & 1.18 & 1 \\
2 & 9961 & 4770 & 1.43 & 2.56 & 1 \\
\hline
\end{tabular}
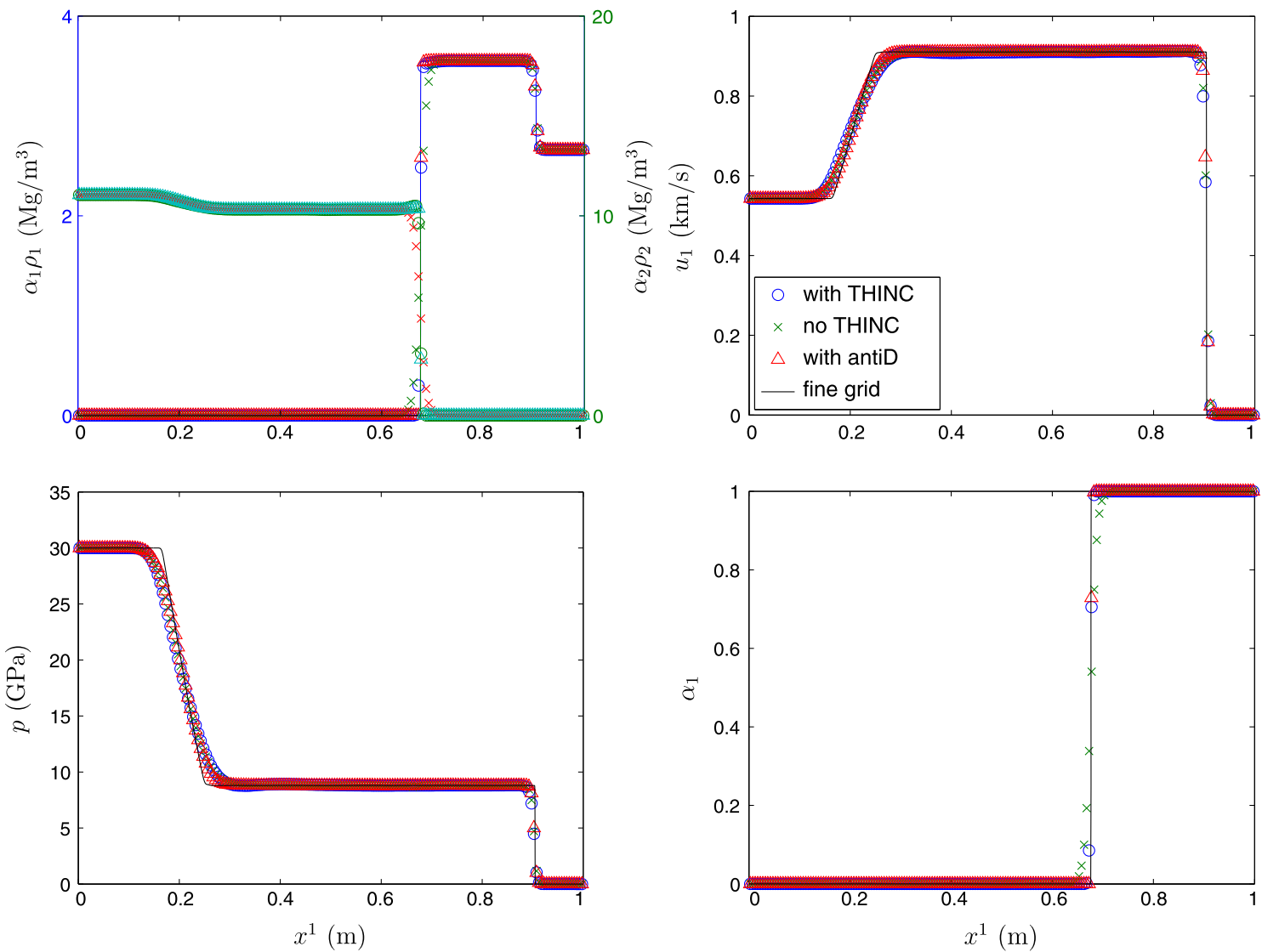

Fig. 3. Numerical results for a shock wave in molybdenum interacting with an encapsulated MORB liquid at time $120 \mu$. The graphs are displayed in the same manner as in Fig. 2.

Here $\Gamma_{0 k}=\gamma_{k}-1$ represents a reference Mie-Grüneisen coefficient at $\rho_{k}=\rho_{0 k}, \eta_{k} \in[0,1]$ is a dimensionless parameter, $c_{0 k}$ denotes the zero-pressure isentropic speed of sound, and $\zeta_{k}$ is a dimensionless parameter. With the zero initial reference state for $p_{0}$ and $e_{0}$, typical set of material quantities for the $\operatorname{MORB}(k=1)$ and molybdenum $(k=2)$ are given in Table 2 .

In this problem, the initial condition is composed of a stationary (MORB-molybdenum) interface at $x^{1}=0.6 \mathrm{~m}$ and a rightward going Mach 1.163 shock wave in molybdenum at $x^{1}=0.4 \mathrm{~m}$ traveling from left to right in a shock tube of unit length. The material on the right of the interface is a MORB liquid with the data

$$
\left(\rho_{1}, \rho_{2}, u_{1}, p, \alpha_{1}\right)_{R}=\left(\rho_{01}, \rho_{02}, 0,0,1-10^{-6}\right),
$$

and the material on the left of the interface (i.e., on the middle and the preshock state), is molybdenum with data

$$
\left(\rho_{1}, \rho_{2}, u_{1}, p, \alpha_{1}\right)_{M}=\left(\rho_{01}, \rho_{02}, 0,0,10^{-6}\right) .
$$

The state behind the shock in the molybdenum is

$$
\left(\rho_{1}, \rho_{2}, u_{1}, p, \alpha_{1}\right)_{L}=\left(\rho_{01}, 11042 \mathrm{~kg} / \mathrm{m}^{3}, 543 \mathrm{~m} / \mathrm{s}, 3 \times 10^{10} \mathrm{~Pa}, 10^{-6}\right) .
$$

We note that this gives us one example, in which the (MORB-molybdenum) interface is accelerated by a shock wave coming from the heavy-fluid to the light-fluid region, and it is known that the resulting wave pattern after the interaction would consist of a transmitted shock wave, an interface, and a reflected rarefaction wave (cf. [10]).

Numerical results for this problem are shown in Fig. 3, where the snap shot of density, velocity, pressure, and volume fraction for MORB are shown at time $t=120 \mu$ s using a 200 grid. From the figure, we again observe sensible improvement 
Table 3

Material quantities in cgs units for gaseous explosive $(k=1)$ and water $(k=2)$ in the JWL equation of state (22) for a spherical underwater explosion test.

\begin{tabular}{llcccc}
\hline$k$ & $\rho_{0 k}\left(\mathrm{~g} / \mathrm{cm}^{3}\right)$ & $\mathcal{A}_{1 k}\left(\mathrm{dyn} / \mathrm{cm}^{2}\right)$ & $\mathcal{A}_{2 k}\left(\mathrm{dyn} / \mathrm{cm}^{2}\right)$ & $\mathcal{R}_{1 k}$ & $\mathcal{R}_{2 k}$ \\
\hline 1 & 1.63 & $3.712 \times 10^{12}$ & $3.23 \times 10^{10}$ & 4.15 & 0.95 \\
2 & 1.00381 & $15.82 \times 10^{12}$ & $-4.668 \times 10^{10}$ & 1.3 & 8.94
\end{tabular}

of the interface structure obtained using our THINC-based method; this result is comparable with the anti-diffusion one in all region, except in a narrow region on the left of the interface as in the previous case where a slight overshoot on $\alpha_{1} \rho_{1}$ is present. Despite this, our THINC scheme works in a satisfactory manner for the other physical variables near the interfaces. A two-dimensional version of this problem will be considered in Example 5.8 .

Example 5.4. To end this subsection, we are interested in a well-studied UNDEX (UNDerwater EXplosion) problem that involves the growth and collapse of an underwater gas bubble in spherically symmetric geometry. Similar to the test case considered in [25] (see [58] also), the initial condition we take is composed of $300 \mathrm{~g}$ of spherical TNT charge at a depth of $94.1 \mathrm{~m}$ in water with ambient pressure of $1 \times 10^{7} \mathrm{dyn} / \mathrm{cm}^{2}$. In this work, the constitutive law for both the explosive $(k=1)$ and water $(k=2)$ are described by the JWL (Jones-Wilkins-Lee) equation of state where in (2) we have

$$
\begin{aligned}
& \Gamma_{k}=\gamma_{k}-1, \\
& p_{\infty, k}\left(\rho_{k}\right)=\mathcal{A}_{1 k} \exp \left(\frac{-\rho_{0 k} \mathcal{R}_{1 k}}{\rho_{k}}\right)+\mathcal{A}_{2 k} \exp \left(\frac{-\rho_{0 k} \mathcal{R}_{2 k}}{\rho_{k}}\right), \\
& e_{\infty, k}\left(\rho_{k}\right)=\frac{\mathcal{A}_{1 k}}{\rho_{0 k} \mathcal{R}_{1 k}} \exp \left(\frac{-\rho_{0 k} \mathcal{R}_{1 k}}{\rho_{k}}\right)+\frac{\mathcal{A}_{2 k}}{\rho_{0 k} \mathcal{R}_{2 k}} \exp \left(\frac{-\rho_{0 k} \mathcal{R}_{2 k}}{\rho_{k}}\right),
\end{aligned}
$$

see Table 3 for a sample set of material quantities in cgs units for TNT and water (cf. [58]). With that, inside the explosive sphere of radius $3.5287 \mathrm{~cm}$ (for a $300 \mathrm{~g}$ TNT) the state variables are

$$
\left(\rho_{1}, \rho_{2}, u_{1}, p, \alpha_{1}\right)=\left(\rho_{01}, \rho_{02}, 0,83812408875.7788 \text { dyn, } 1-10^{-8}\right),
$$

and outside the sphere the state variables are

$$
\left(\rho_{1}, \rho_{2}, u_{1}, p, \alpha_{1}\right)=\left(\rho_{01}, \rho_{02}, 0,1 \times 10^{7} \text { dyn, } 10^{-8}\right) .
$$

It should be mentioned that unlike the work done in $[25,58]$ where a hybrid barotropic and non-barotropic equation of state (i.e., the Tait equation of state for water, and the JWL for TNT) is used in their numerical simulation of the problem with ALE-type methods, here with the five-equation model (4) and the definition of mixture pressure (3) it is convenient to consider a non-barotropic equation of state for water (such as the JWL) as well in our numerical method for simulation. It has been demonstrated in [58] and the results shown below that the influence on the equation of state for water is not high for this spherical UNDEX problem; the compressibility effect of water counts however.

We carry out the computation using the THINC-based interface-sharpening method proposed here together with the source term treatment described in Section 2.3 for spherical symmetry, where the solid wall is used on the left boundary, and the non-reflection boundary is used on the right. As in the results present in [25], Figs. 4(a)-(d) show the time sequence of the density and pressure through four different solution phases, namely, the initial phase, the shock-interface wave interaction phase, the incompressible phase, and bubble collapse and rebound phase, respectively. First, note that we have used an adaptive domain size, i.e., 20,60,160, and $5000 \mathrm{~cm}$ for the solution phases (a)-(d) individually, so that the leading shock wave remains in the computational domain; the mesh size is kept as a constant $\Delta x^{1}=0.05 \mathrm{~cm}$ throughout the runs. Comparing our results with the ones shown in [25], we see a qualitative agreement on the basic structure of the solution at all the phases.

To give a quantitative assessment of the solutions for this spherical UNDEX problem, Fig. 5 shows the time history of the bubble radius and the bubble-water interface pressure obtained using both the THINC- and anti-diffusion-based methods (cf. $[11,25,58]$ ). From the figure, it is easy to estimate the maximum bubble radius (denoted by $r_{\text {max }}$ ) by checking the maximum of the discrete set of the bubble radius. Moreover, we may also estimate the bubble period (denoted by $T_{b}$ ) by measuring either the minimum radius or the peak interface after the maximum volume expansion. Table 4 summarizes the findings together with those results reported in the literature as for comparison.

Note that the incompressible solution shown in the table is computed based on solving a variant of the RayleighPlesset equation with the gas pressure on the bubble surface following the isentropic JWL equation of state, see [36,58] for the details. From the table, it is clear that the incompressible solution does not give a good prediction on the solution which indicates that the water compressibility is of fundamental importance to this problem. Comparing our results with those appeared in the literature (cf. $[52,25,58]$ ) and the anti-diffusion ones, we observe good agreement of results. 
(a)

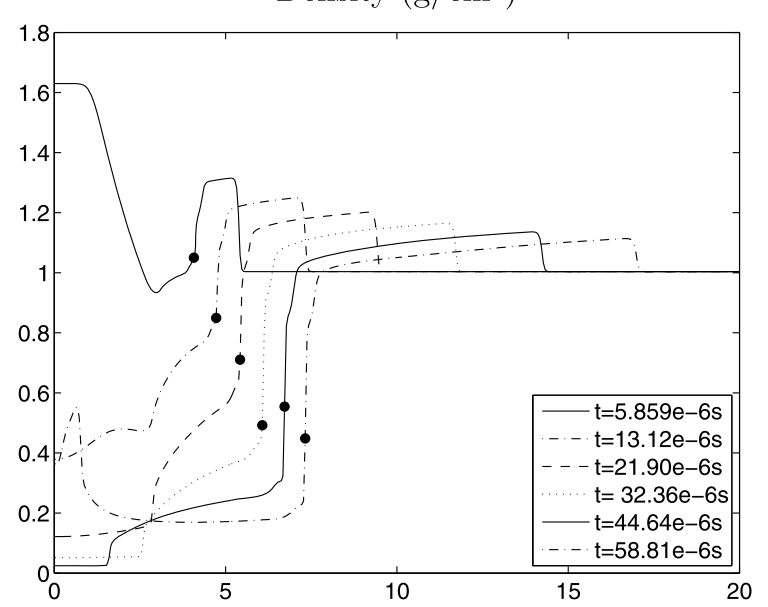

(b)

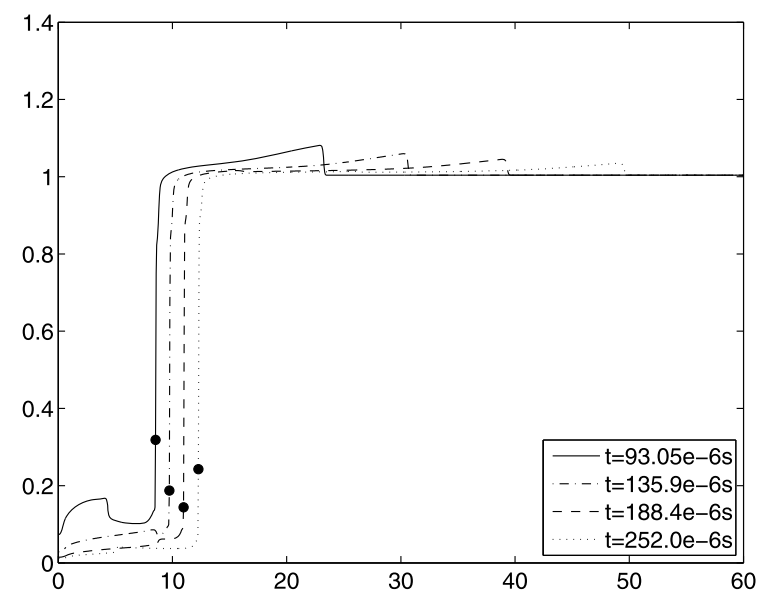

(c)

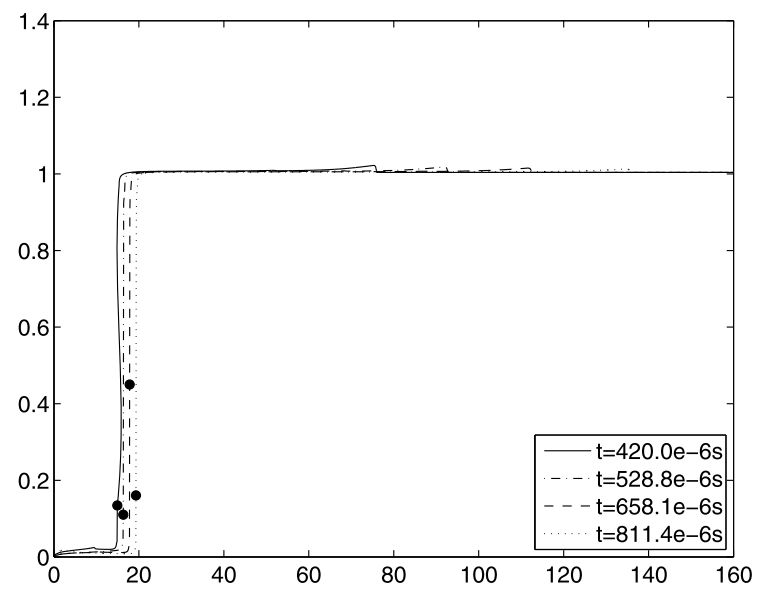

Pressure $\left(\mathrm{dyn} / \mathrm{cm}^{2}\right)$
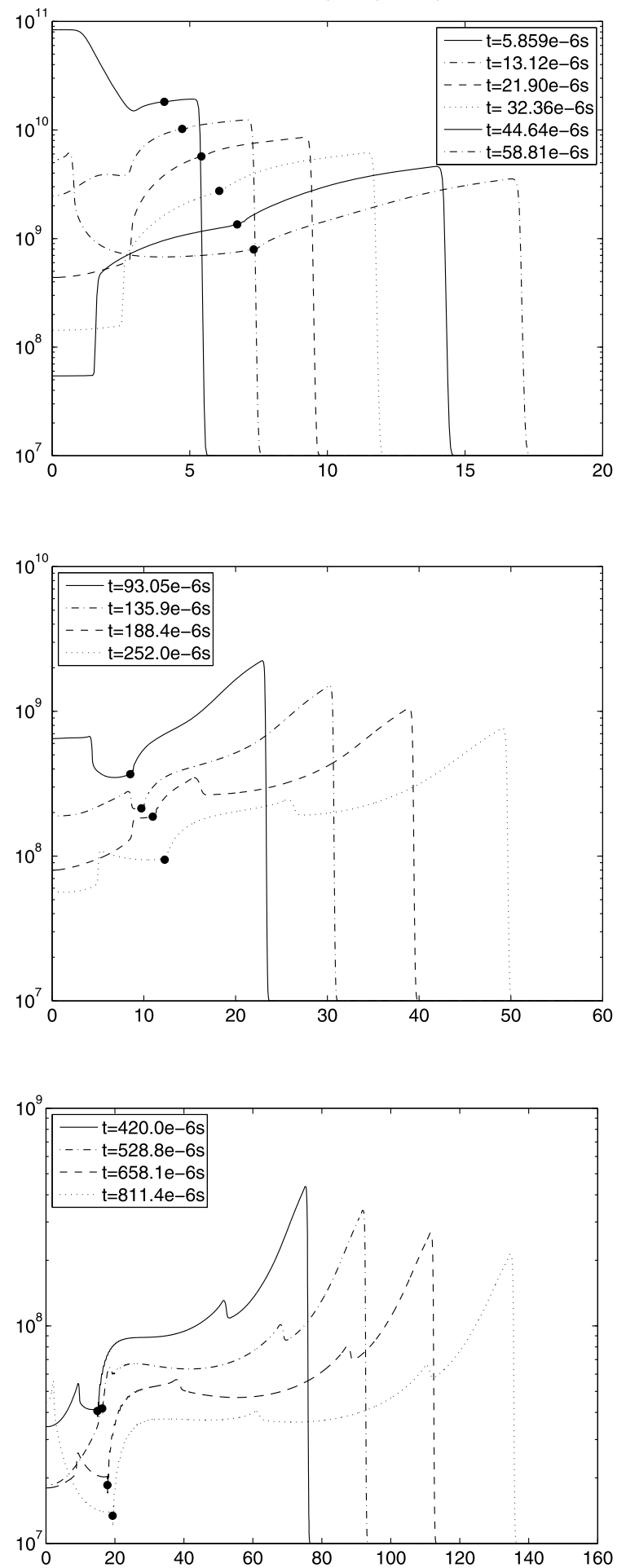

Fig. 4. Numerical results for a spherical UNDEX test. Snap shots of the density and pressure are shown at four different solution phases, namely, from top row to bottom, they are at (a) the initial phase, (b) the shock-interface wave interaction phase, (c) the incompressible phase, and (d) bubble collapse and rebound phase. The solid dots plotted in each graph indicate the approximate solution of the gas-water interface. 
(d)

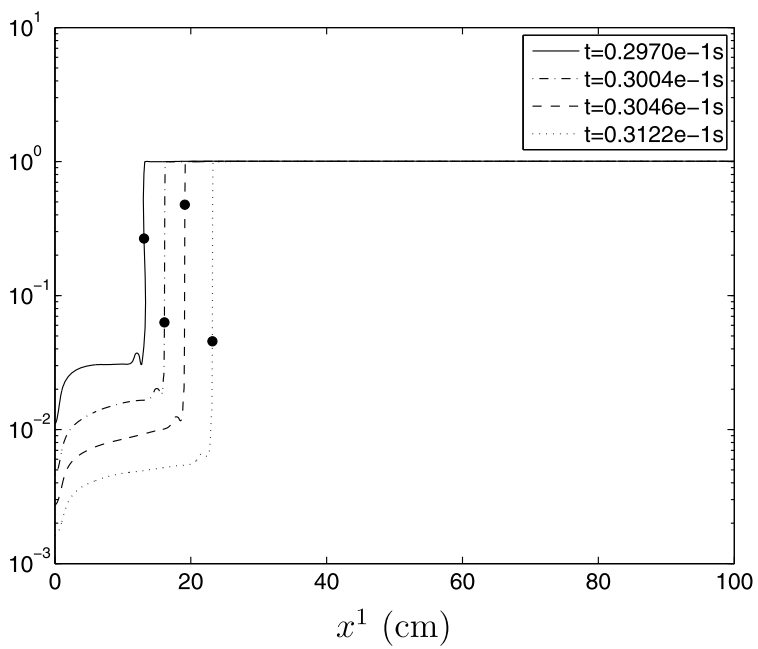

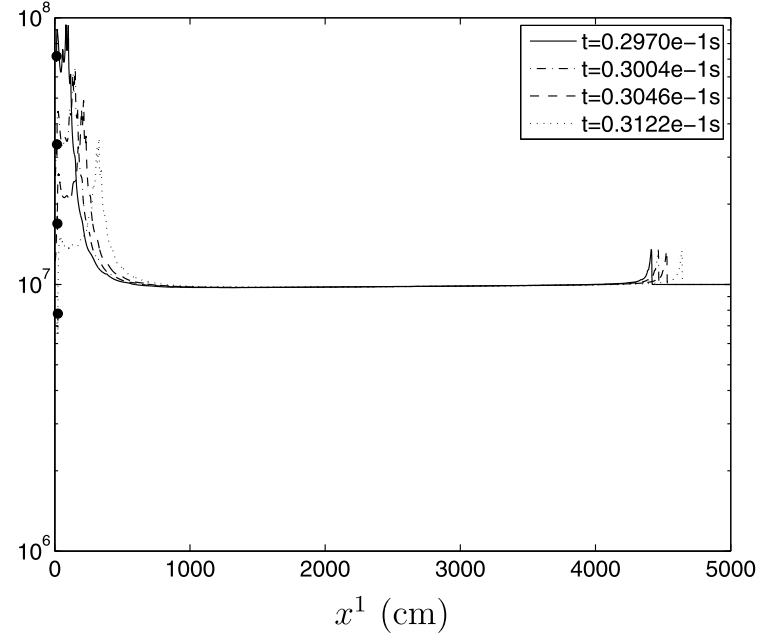

Fig. 4. (continued)
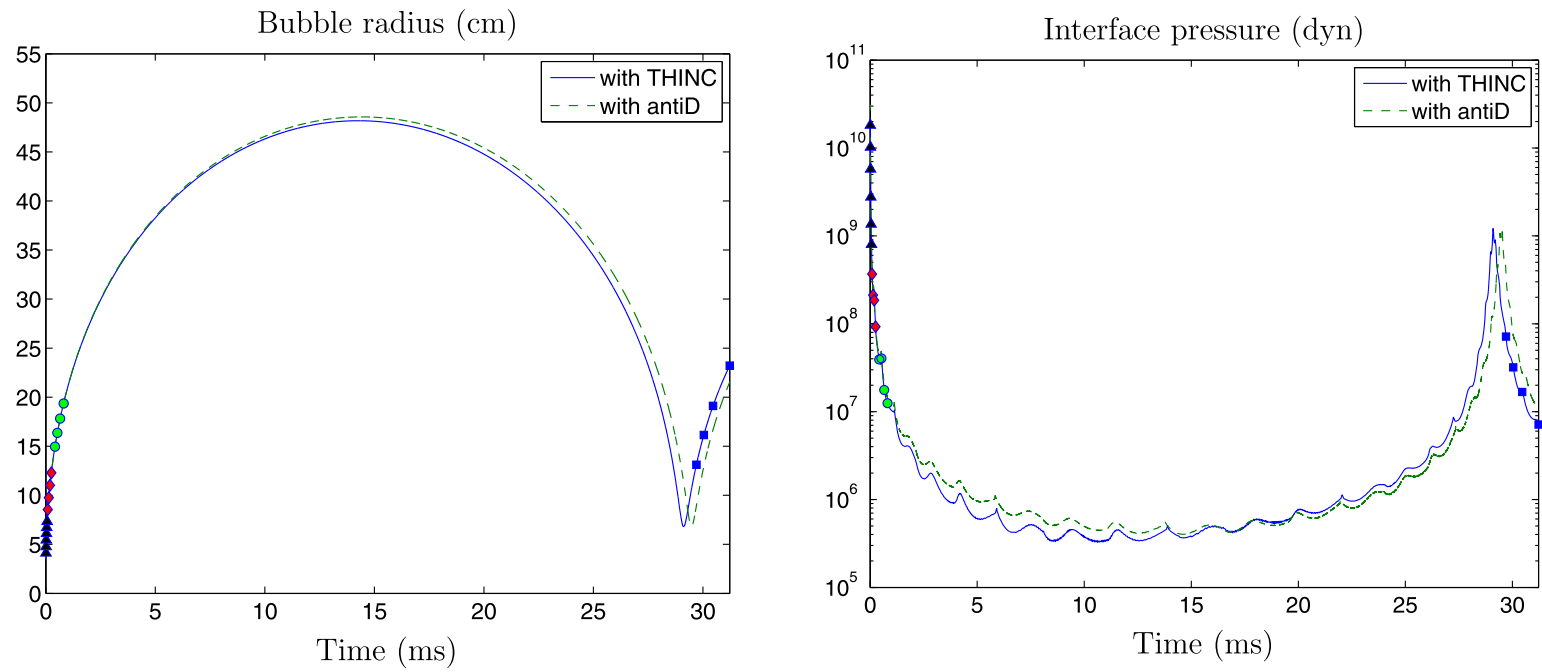

Fig. 5. Time history of the bubble radius and the bubble-water interface pressure for the spherical UNDEX test. The black triangles, red diamonds, green circles, and blue squares, shown in each graph indicate the selected snap-shot time plotted in Fig. 4(a)-(d), respectively. (For interpretation of the references to color in this figure legend, the reader is referred to the web version of this article.)

Table 4

A quantitative study of the maximum bubble radius $r_{\max }$ and the period of bubble oscillation $T_{b}$ for the spherical underwater explosion test.

\begin{tabular}{|c|c|c|c|c|}
\hline & $r_{\max }(\mathrm{cm})$ & Error (\%) & $T_{b}(\mathrm{~ms})$ & Error (\%) \\
\hline Experiment [52] & 48.10 & 0 & 29.8 & 0 \\
\hline Incompressible & 66.49 & 38.2 & 39.1 & 31.2 \\
\hline Luo et al. [25] & 48.75 & 1.4 & 29.7 & 0.3 \\
\hline Wardlaw et al. [58] & 46.40 & 3.5 & 29.8 & 0 \\
\hline Our results (with THINC) & 48.17 & 0.1 & 29.1 & 2.3 \\
\hline Our results (with antiD) & 48.57 & 0.1 & 29.5 & 1.1 \\
\hline
\end{tabular}

It should be mentioned that this spherical UNDEX problem is known to be a difficult test for any multi-material solvers [25] as the flow conditions are extreme, and the equation of states are stiff. Any spurious oscillations on solution states near the bubble-water interface will lead eventually to unphysical thermodynamic states, yielding the collapse of computations; this is in fact the case we have experienced when the original semi-discrete method without THINC reconstruction is employed to solve the problem. The computed UNDEX solutions presented here have demon- 
With THINC

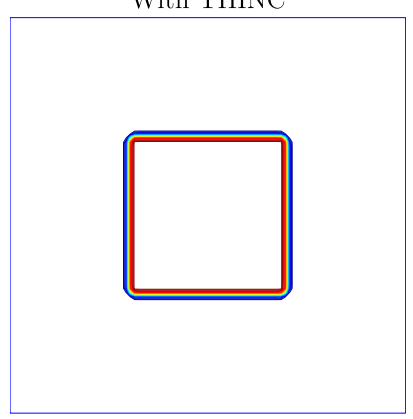

No THINC

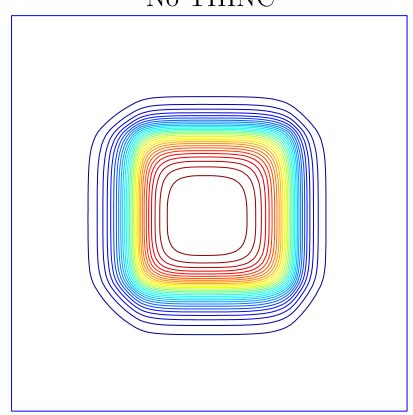

Fig. 6. Numerical results for a passive evolution of a square liquid column in gas. Contours of the density are shown at time $t=20 \mathrm{~ms}$ obtained using the method with and without THINC reconstruction on a $100 \times 100$ grid.

Table 5

A comparison of 1-norm errors in mixture density for the passive advection problem obtained using the semi-discrete method with and without THINC reconstruction, and the wave propagation method with anti-diffusion.

\begin{tabular}{|c|c|c|c|c|c|c|}
\hline \multirow[t]{3}{*}{$N$} & \multicolumn{6}{|l|}{ Method } \\
\hline & \multicolumn{2}{|c|}{ With THINC } & \multicolumn{2}{|c|}{ No THINC } & \multicolumn{2}{|c|}{ With anti-diffusion } \\
\hline & $\overline{\mathcal{E}_{1}(\rho)}$ & Order & $\overline{\mathcal{E}_{1}(\rho)}$ & Order & $\overline{\mathcal{E}_{1}}(\rho)$ & Order \\
\hline 50 & 9.8840 & $\mathrm{NaN}$ & 91.7486 & $\mathrm{NaN}$ & 4.0436 & $\mathrm{NaN}$ \\
\hline 100 & 5.1746 & 0.93 & 60.6698 & 0.60 & 2.0558 & 0.98 \\
\hline 200 & 2.6455 & 0.97 & 39.3623 & 0.62 & 0.9921 & 1.05 \\
\hline 400 & 1.3373 & 0.98 & 25.2699 & 0.64 & 0.4414 & 1.17 \\
\hline
\end{tabular}

strated further the viability of the proposed interface-sharpening method for practical compressible two-phase flow problems.

\subsection{Two-dimensional case}

Example 5.5. To show how our method works in two dimensions, we begin by considering a simple interface only problem in a unit square domain where the exact solution consists of a passive evolution of a square liquid column in gas of size $\left(x^{1}, x^{2}\right) \in[0.3,0.7] \times[0.3,0.7] \mathrm{m}^{2}$ with uniform equilibrium pressure $p=10^{5} \mathrm{~Pa}$ and constant particle velocity $\left(u_{1}, u_{2}\right)=$ $(100,100) \mathrm{m} / \mathrm{s}$. In this test, inside the square region $\left(x^{1}, x^{2}\right) \in[0.3,0.7] \times[0.3,0.7] \mathrm{m}^{2}$ the fluid is nearly liquid that contains a uniformly distributed small amount of the gas volume fraction $\alpha_{2}=10^{-8}$, and it is nearly gas that contains the liquid volume fraction $\alpha_{1}=10^{-8}$ elsewhere; the density for the pure liquid and gas phase in the domain are $\rho_{1}=1000 \mathrm{~kg} / \mathrm{m}^{3}$ and $\rho_{2}=1 \mathrm{~kg} / \mathrm{m}^{3}$, respectively. We use the linearized Mie-Grüneisen equation of state to model the liquid and gas where the material-dependent functions appeared in (2) are $\Gamma_{k}=\gamma_{k}-1, p_{\infty, k}\left(\rho_{k}\right)=c_{0 k}^{2}\left(\rho_{k}-\rho_{0 k}\right)$, and $e_{\infty, k}=0$ with the parameter values taken in turn to be $\gamma_{1}=4.4, c_{01}=1624.8 \mathrm{~m} / \mathrm{s}, \rho_{01}=1000 \mathrm{~kg} / \mathrm{m}^{3}$, and $\gamma_{2}=1.4, c_{02}=0, \rho_{02}=1 \mathrm{~kg} / \mathrm{m}^{3}$. Here periodic boundary condition is used in all sides.

Fig. 6 shows contour plots of the density obtained using the method with and without THINC reconstruction at time $t=20 \mathrm{~ms}$ (this is the time the water column traveled over two periodic distance of the domain) using a $100 \times 100$ grid. Excellent interface-sharpening result is observed when THINC reconstruction is employed in the method, whereas severely diffused interface is seen otherwise. It is not difficult to show analytically that both the pressure and the velocity retains their equilibrium state, see Section 4.2 as an example.

In Table 5, we present numerical results for a convergence study of the 1-norm errors in mixture density, denoted by $\mathcal{E}_{1}(\rho)$, as the mesh is refined (cf. [21]). We note that the method is first order accurate when the interface-sharpening technique (i.e., with either the THINC- or anti-diffusion-based) is in use, and is less than first order accurate otherwise; this rate of convergence is true also for the 1-norm errors in volume fraction considered in this case (not shown here).

Example 5.6. Our second test problem in two dimensions is a three state two-phase triple point problem. We use the same problem setup as considered in [19,24], where in a rectangular domain of size $\left(x^{1}, x^{2}\right) \in[0,7] \times[0,3]$ the initial data is quiescent with the state variables

$$
\left(\rho_{1}, \rho_{2}, p, \alpha_{1}\right)= \begin{cases}\left(1,1,1,1-10^{-8}\right) & \text { if } x^{1}<1 \\ \left(1,1,0.1,10^{-8}\right) & \text { if } x^{1}>1 \text { and } x^{2}<1.5 \\ \left(0.125,1,0.1,1-10^{-8}\right) & \text { if otherwise }\end{cases}
$$



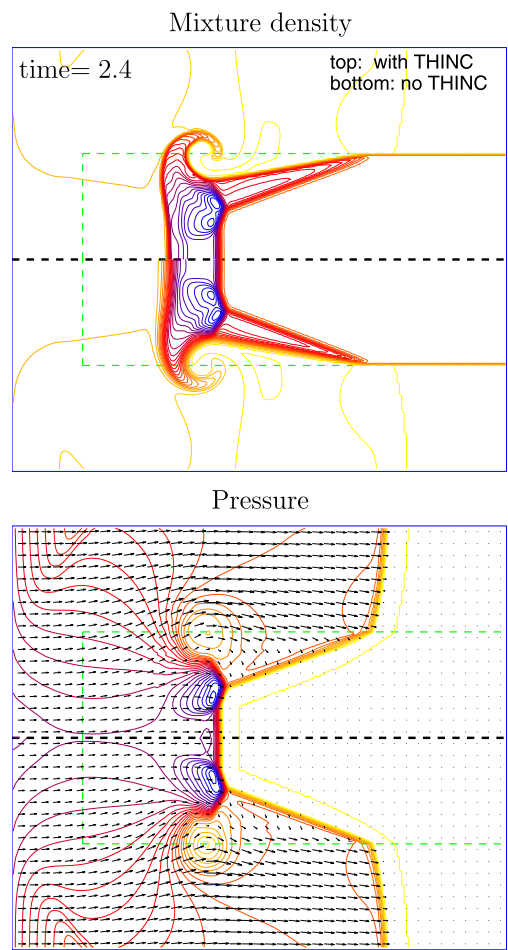

Volume fraction

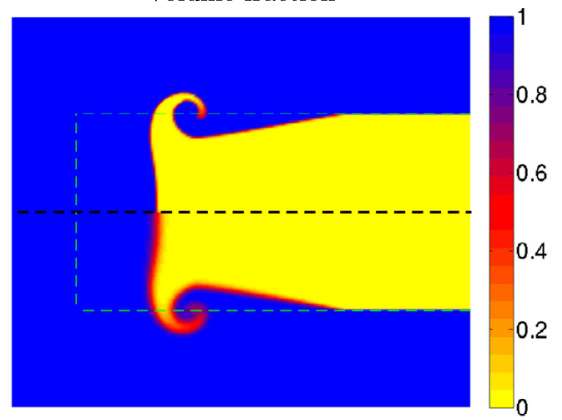

Mixture density

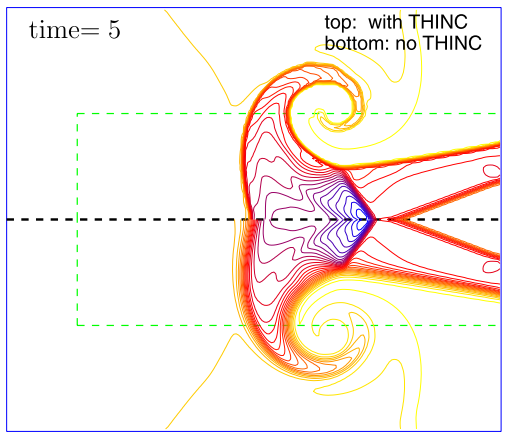

Pressure

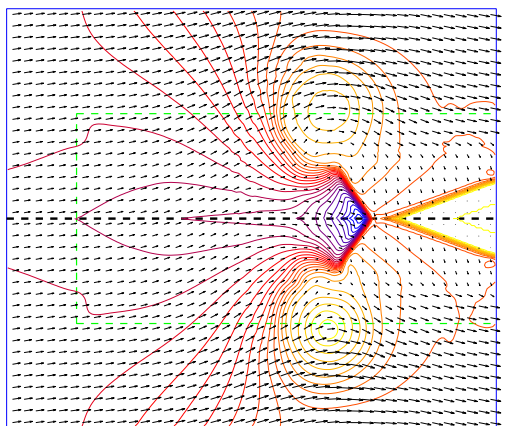

Volume fraction

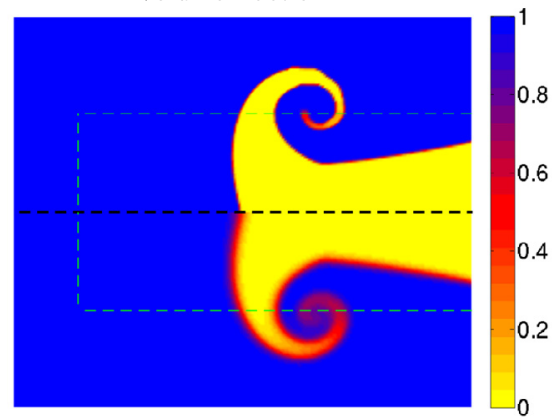

Fig. 7. Numerical results for a three state two-phase triple point problem. Contours of mixture density, pressure, and pseudo colors of volume fraction are shown at two different times $t=2.4$ and 5 obtained using each of the methods with and without THINC interface-sharpening (drawn on the top and bottom parts of each graph, respectively) with a $210 \times 90$ grid. The vector fields shown in the pressure plots are the particle velocities, and the green dashed-line graphed in each plot is the initial location of the interface. (For interpretation of the references to color in this figure legend, the reader is referred to the web version of this article.)

Here the two different fluid phases inside the domain are all modeled by polytropic perfect gases so that in (2) we have $\Gamma_{k}=\gamma_{k}-1, p_{\infty, k}=0$, and $e_{\infty, k}=0$. We take $\gamma_{1}=1.5$ and $\gamma_{2}=1.4$ for phase 1 and 2, respectively, see Fig. 7 for the initial location of the material interface. Nonreflecting boundary condition is assumed on all sides for this problem.

With that, it is known that due to the pressure difference at $x^{1}=1$, a planarly shock wave is formed moving to the right. Because of the different material properties on the right where the acoustic impedance in upper part is greater than the one in the lower part, the shock wave moves faster in the upper, and so a vortex evolves around the triple point as time proceeds.

As before, this triple point problem is solved using each of the methods with and without interface sharpening. The results are shown in Fig. 7, where contours of density, pressure, and pseudo colors of volume fraction are plotted at two different times $t=2.4$ and 5 with a $210 \times 90$ grid and the vector fields of the particle velocities are included in the pressure plots also. From the figure, it is clear that both methods have done a reasonable job in capturing the main feature of the solution without introducing any spurious oscillation in pressure near the interface (cf. [19,24]). It is interesting to see that the results obtained using our interface-sharpening method give a sharper resolution of the interface, while maintaining the same structure of the solution away from the interface, which follow our expectations in the development of this method. For comparison purposes, Fig. 8 shows numerical results for the same run but is with the anti-diffusion method, observing good agreement with the THINC results qualitatively. 

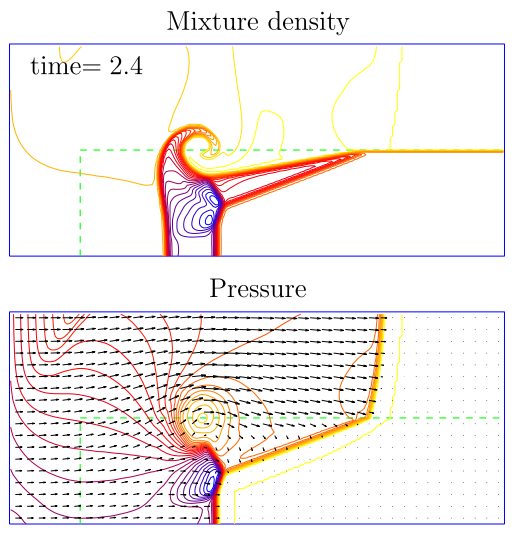

Volume fraction

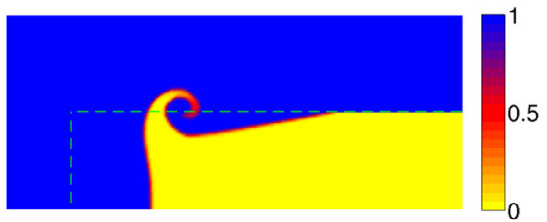

Mixture density

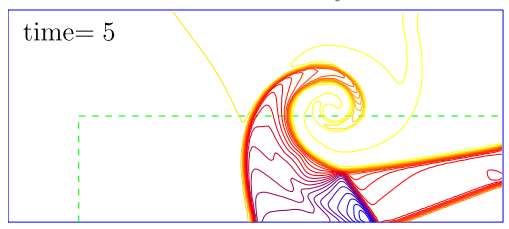

Pressure

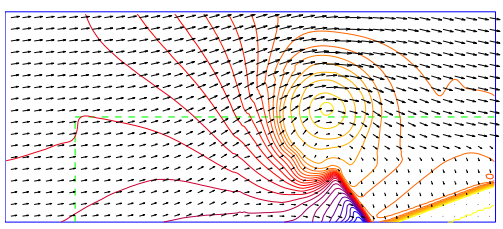

Volume fraction

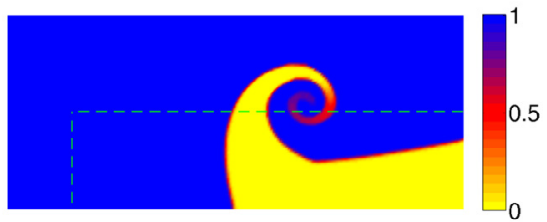

Fig. 8. Anti-diffusion results for a three state two-phase triple point problem. Contours of mixture density, pressure, and pseudo colors of volume fraction are shown at two different times $t=2.4$ and 5 with a $210 \times 90$ grid.

Example 5.7. We are next concerned with a well-known shock-bubble interaction problem that involves the collision of a shock wave in air with a circular R22 gas bubble (cf. [8,18,28,37,45] and cited references therein). Initially, there is a planar leftward-moving Mach 1.22 shock wave in air located at $x=275 \mathrm{~mm}$ traveling towards a stationary R22 gas bubble with center $\left(x_{0}^{1}, x_{0}^{2}\right)=(225,44.5) \mathrm{mm}$ and of radius $r_{0}=25 \mathrm{~mm}$ lying in front of it. Here both the air and R22 are modeled as perfect gases with $\gamma_{1}=1.249, \rho_{01}=3.863 \mathrm{~kg} / \mathrm{m}^{3}$ and $\gamma_{2}=1.4, \rho_{02}=1.225 \mathrm{~kg} / \mathrm{m}^{3}$ for R22 $(k=1)$ and air $(k=2)$, respectively. Inside the $\mathrm{R} 22$ gas bubble, the state variables are

$$
\left(\rho_{1}, \rho_{2}, u_{1}, u_{2}, p, \alpha_{1}\right)=\left(\rho_{01}, \rho_{02}, 0,0,1.01325 \times 10^{5} \mathrm{~Pa}, 1-10^{-8}\right),
$$

while outside the bubble they are

$$
\left(\rho_{1}, \rho_{2}, u_{1}, u_{2}, p, \alpha_{1}\right)=\left(\rho_{01}, \rho_{02}, 0,0,1.01325 \times 10^{5} \mathrm{~Pa}, 10^{-8}\right)
$$

and

$$
\left(\rho_{1}, \rho_{2}, u_{1}, u_{2}, p, \alpha_{1}\right)=\left(\rho_{01}, 1.686 \mathrm{~kg} / \mathrm{m}^{3},-113.5 \mathrm{~m} / \mathrm{s}, 0,1.59 \times 10^{5} \mathrm{~Pa}, 10^{-8}\right)
$$

in the preshock and postshock regions, respectively. The computational domain considered here is a shock tube of size: $\left(x^{1}, x^{2}\right) \in[0,445] \times[0,89] \mathrm{mm}^{2}$, where we impose the solid wall boundary condition on the top and bottom, and the non-reflecting boundary condition on the left and right.

Fig. 9 shows a sequence of the schlieren-type images of the density at eight different times $t=55,115,187,247,318$, 342,417 , and $1020 \mu$ s (measured relative to the time where the incident shock first hits the upstream bubble wall) with a $3560 \times 356$ grid. Here for clarity, only the results in some short distances around the gas bubble are presented. From the figure, it is easy to observe the sensible improvement on the sharpness of the interface when the interface-sharpening version of the method is employed in the runs.

In Fig. 10, we show density schlieren-type images for the anti-diffusion run of this problem; see the results shown in [51] also. With that, it is interesting to see that we have gotten a more regularized interface structure (see the results with THINC in Fig. 9) than the one obtained using the anti-diffusion method. This comes as no surprise, however, because in our interface-sharpening method the THINC reconstruction is applied only to interface cells where the monotonicity constraint (13) is one of the conditions to be satisfied, while in the other instance, the anti-diffusion step is applied to all the cells with a numerical regularization condition imposed to stabilize the gradient of volume fraction in discretization.

To get a quantitative assessment of several prominent features of the flow, following [37,45] we make a diagnosis plot of the space-time locations of the incident shock wave, the upstream bubble wall, the downstream bubble wall, the refracted shock wave, and the transmitted shock wave at some selected times up to $t=250 \mu \mathrm{s}$, see Fig. 11 for the case with and without the THINC reconstruction. With these trajectories, we perform a linear least-squares fit to each set of points separately, and take the slope of the respective line as one measure of the wave speed of interests. Table 6 
time $=55 \mu \mathrm{s}$
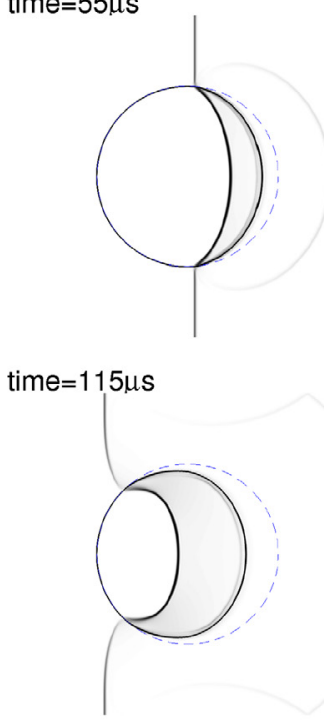

time $=187 \mu \mathrm{s}$

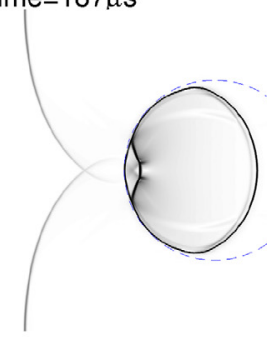

time $=247 \mu \mathrm{s}$

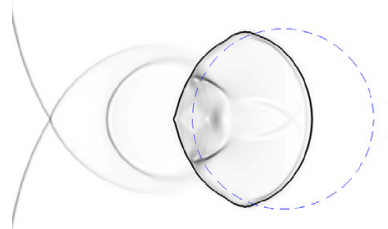

time $=55 \mu \mathrm{s}$
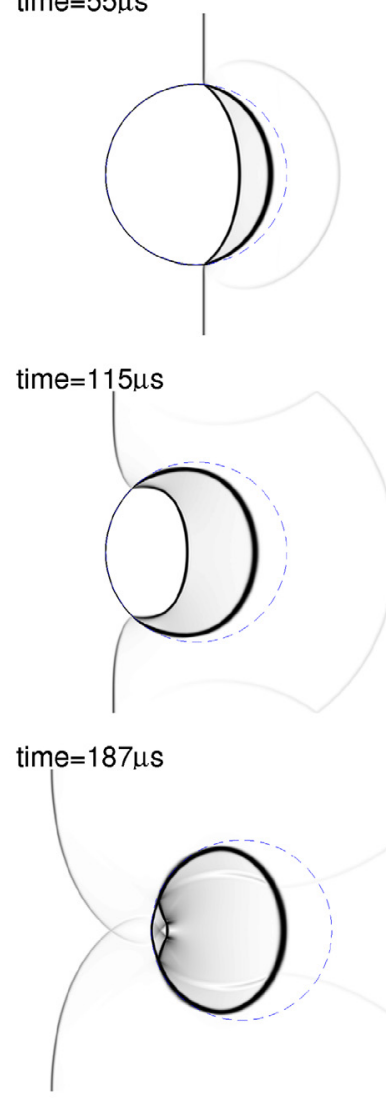

time $=247 \mu \mathrm{s}$

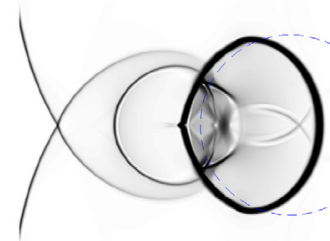

Fig. 9. Numerical results for a planar Mach 1.22 shock wave in air interacting with a circular R22 gas bubble. A sequence of the schlieren-type images of the density obtained using each of the methods with and without THINC reconstruction is shown (from top to bottom) at eight different times $t=55$, 115 , $187,247,318,342,417$, and $1020 \mu$ s with a $3560 \times 356$ grid.

provides a comparison of the various computed velocities between our results with those appeared in the literature, observing good agreement of results. Here $V_{s}$ represents the speed of the incident shock wave during the time $t \in[0,250] \mu$, $V_{u i}\left(V_{u f}\right)$ is the speed of the initial (final) upstream bubble wall when time $t \in[0,400] \mu \mathrm{s}(t \in[400,1000] \mu \mathrm{s}), V_{d i}$ $\left(V_{d f}\right)$ is the speed of the initial (final) downstream bubble wall when time $t \in[200,400] \mu s(t \in[400,1000] \mu s), V_{R}$ is the speed of the refracted shock when time $t \in[0,202] \mu \mathrm{s}$, and $V_{T}$ is the speed of the transmitted shock when time $t \in[202,250] \mu \mathrm{s}$.

Example 5.8. To end this section, we consider a simplified test problem proposed by Miller and Puckett [31] in which a shock wave in molybdenum is interacting with a region of encapsulated MORB liquid in a unit square domain; there is no free surface in the problem formulation. Similar to the initial condition used in Example 5.3 , at $x^{1}=0.3 \mathrm{~m}$, there is a planarly rightward-moving Mach 1.163 shock wave in molybdenum traveling from left to right that is about to collide with a rectangular region $[0.4,0.7] \times[0,0.5] \mathrm{m}^{2}$ which contains a MORB liquid inside. As before, we use the Mie-Grüneisen equation of state (2) with (21) and material parameters given in Table 2 to model the thermodynamic behavior of MORB and molybdenum. Here the solid wall boundary condition is used on the bottom, and the nonreflecting boundary condition is used on the remaining sides. 
With THINC

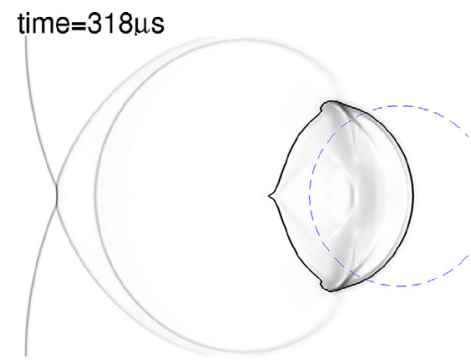

time $=342 \mu \mathrm{s}$

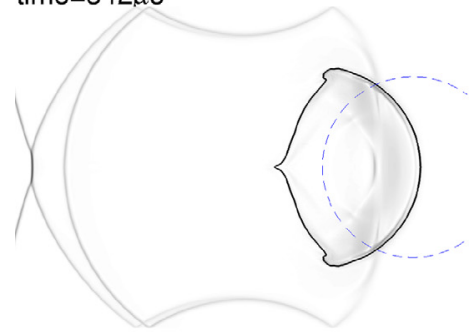

time $=417 \mu \mathrm{s}$

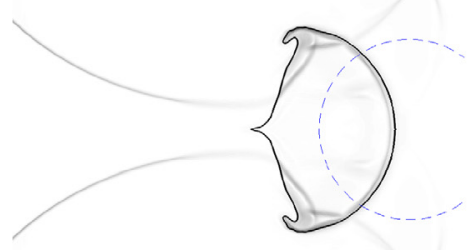

time $=1020 \mu \mathrm{s}$

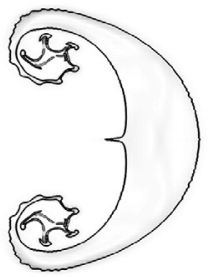

No THINC
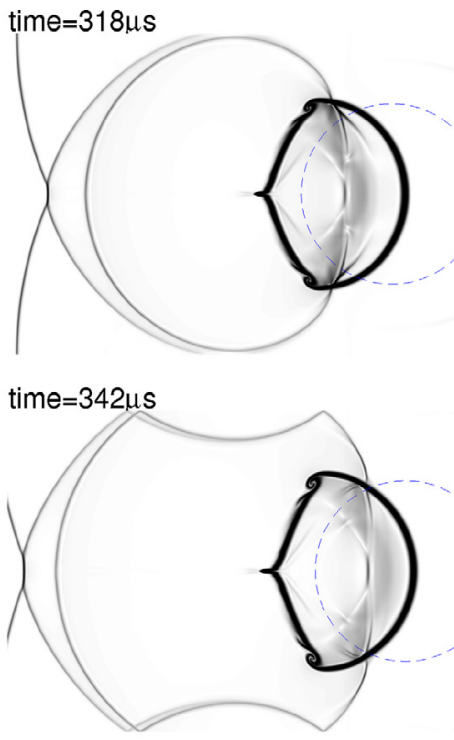

time $=417 \mu \mathrm{s}$

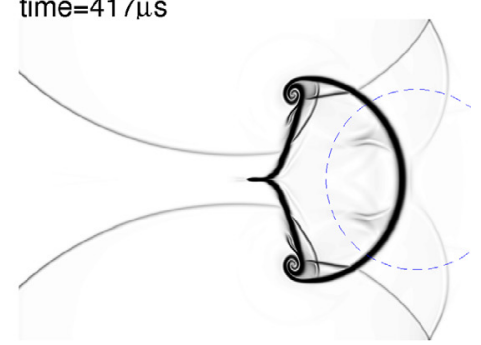

time $=1020 \mu \mathrm{s}$

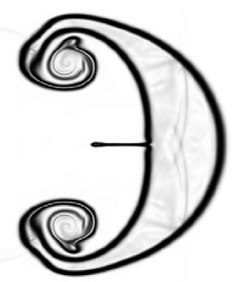

Fig. 9. (continued)

Table 6

A comparison of the computed velocities obtained using our algorithm for Example 5.7 with those reported in the literature; see the text for the definition of the notations used in the table.

\begin{tabular}{|c|c|c|c|c|c|c|c|}
\hline Velocity (m/s) & $V_{s}$ & $V_{R}$ & $V_{T}$ & $V_{u i}$ & $V_{u f}$ & $V_{d i}$ & $V_{d f}$ \\
\hline Experiment [8] & 415 & 240 & 540 & 73 & 90 & 78 & 78 \\
\hline Quirk and Karni [37] & 420 & 254 & 560 & 74 & 90 & 116 & 82 \\
\hline Kokh and Lagoutiere [18] & 411 & 243 & 525 & 65 & 86 & 86 & 64 \\
\hline Ullah et al. [56] & 410 & 246 & 535 & 65 & 86 & 76 & 60 \\
\hline Shyue [45] (volume tracking) & 411 & 243 & 538 & 64 & 87 & 82 & 60 \\
\hline Our results (no THINC) & 410 & 244 & 536 & 65 & 86 & 98 & 76 \\
\hline Our results (with THINC) & 410 & 244 & 538 & 65 & 86 & 87 & 64 \\
\hline Our results (with antiD) & 410 & 244 & 532 & 64 & 85 & 100 & 78 \\
\hline
\end{tabular}



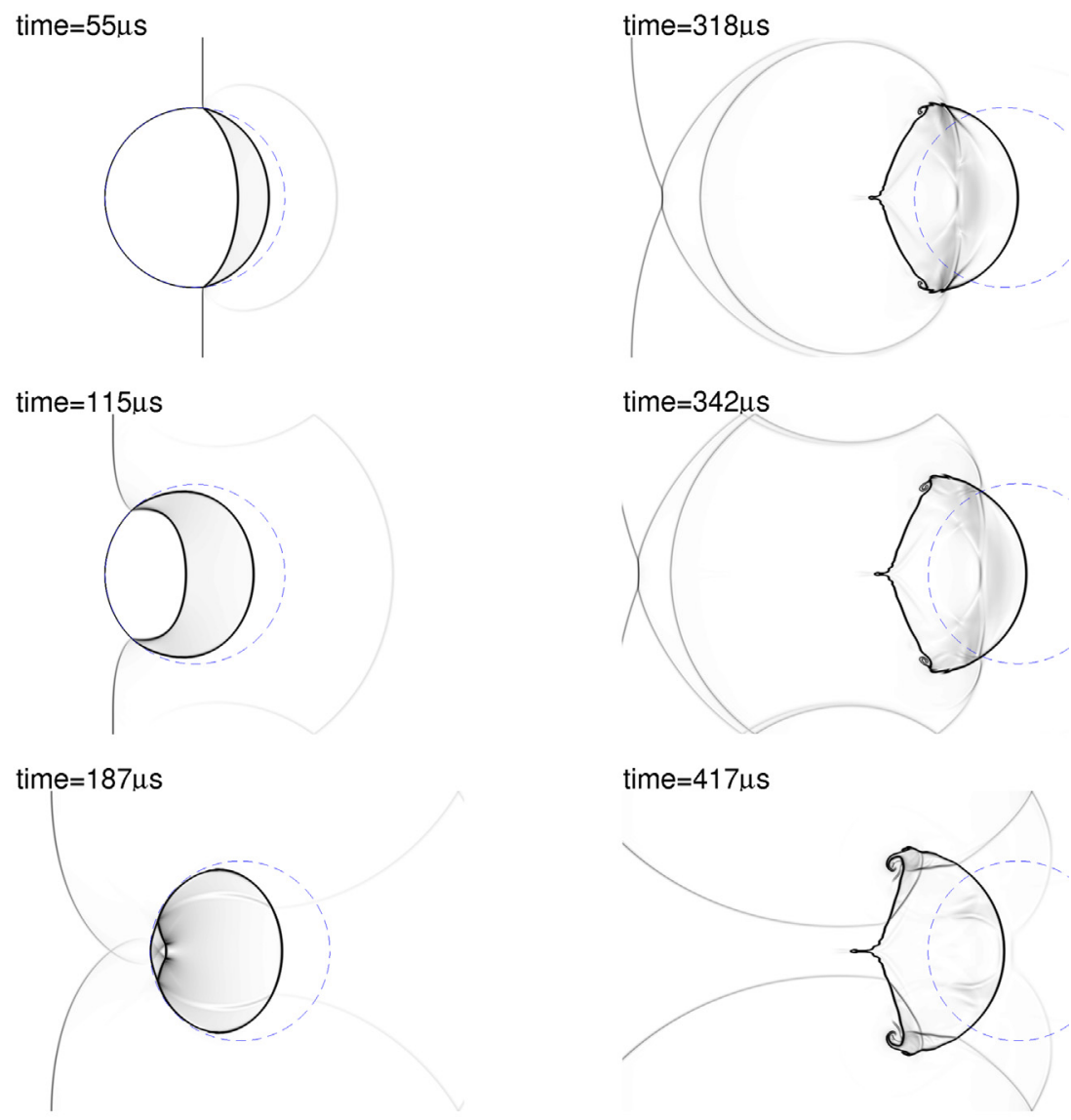

time $=247 \mu \mathrm{s}$

time $=1020 \mu \mathrm{s}$
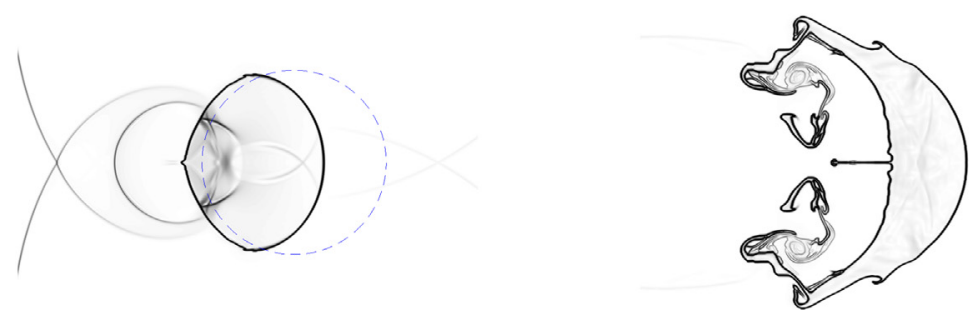

Fig. 10. Anti-diffusion results for a planar Mach 1.22 shock wave in air interacting with a circular R22 gas bubble. Density schlieren-type images are shown at eight different times $t=55,115,187,247,318,342,417$, and $1020 \mu$ sith a $3560 \times 356$ grid.

Fig. 12 show sample results at two different times $t=50$ and $100 \mu$ s obtained using our semi-discrete method with and without THINC reconstruction on a $200 \times 200$ grid. From the pseudo-color plots of the volume fraction, it is easy to observe the improved resolution of the solution near the interface, when the method with THINC reconstruction is employed in the test. In addition to that, from the contours of pressure we find reasonable structure of the diffraction of a shock wave by the MORB liquid, and free of spurious oscillations near the interface. The schlieren-type images of the density provide further evidence on the improvement of the interface structure by our interface-sharpening method, but also show a glitch on the left of the interface. This indicates a slight overshoot of the solution, a numerical artifact that we have already seen in the one-dimensional case. In Fig. 13, we present numerical results based on our implementation of the anti-diffusion method as for comparison, see $[31,43,45,51]$ for a similar test of the problem.

\section{Conclusion}

We have described a simple Eulerian interface-sharpening algorithm for compressible homogeneous two-phase flow governed by a five-equation model. The algorithm uses a semi-discrete wave propagation method as a basis with a variant of the THINC scheme for reconstructing sub-grid discontinuity of volume fractions numerically. The algorithm is very simple 

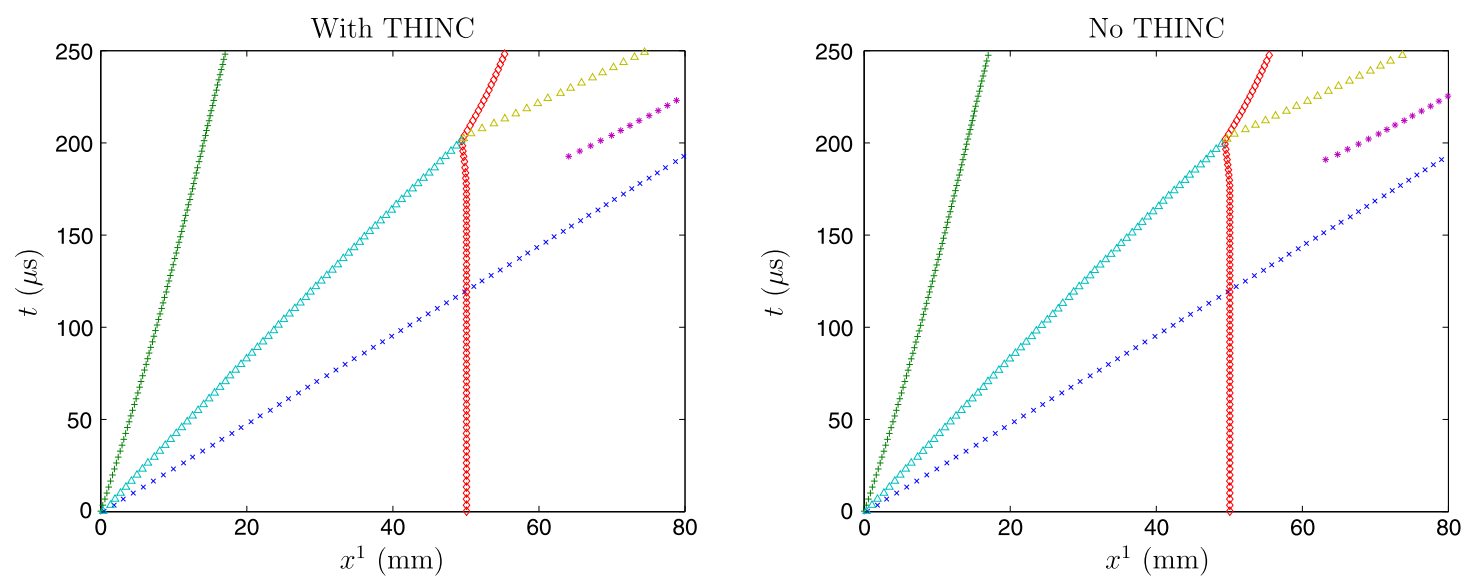

Fig. 11. Space-time locations of the incident shock wave (marked by symbol " $\times$ "), the upstream bubble wall (marked by symbol "+"), the downstream bubble wall (marked by symbol “ $\diamond$ "), the refracted shock (marked by symbol “ $\triangle$ "), and the transmitted shock (marked by symbols “*” and “ $\triangle$ ") for the runs shown in Fig. 9. These trajectories can be used to estimate the speed such as $V_{s}, V_{u}, V_{d}, V_{R}, V_{T}$ in the order mentioned above.

and straightforward as one needs only replace the reconstruction in a conventional finite volume formulation by the THINC reconstruction in the interfacial cell where the volume fraction function has a jump. THINC reconstruction re-enforces the transition jump at every time step and effectively retains the sharpness of the material interface even for long time computations. In spite of its simplicity, the THINC scheme provides a general and effective remedy to remove the numerical diffusion which smears out the jumps in Eulerian formulations. Sample numerical results presented in the paper show the feasibility of the algorithm for sharpening compressible interfaces in one and two dimensions. One ongoing work is to devise a fully multi-dimensional method to high order and also to mapped grids with complex geometries; the fully multi-dimensional THINC reconstruction in [12] can be readily used for that purpose. Extension of the method to a class of liquid-vapor phase transition problems that may be described by a reduced five-equation model of Kapila et al. will be considered also (cf. $[14,40,63])$.

\section{Acknowledgements}

The first author (K.-M. Shyue) was supported in part by the National Science Council Taiwan Grant NSC 101-2115-M-002009 and by the National Center for Theoretical Sciences (Taipei office). The second author (F. Xiao) is partly supported by JSPS KAKENHI (24560187).

\section{Appendix A. Some advection tests}

We present some advection benchmark tests of the volume fraction transport in two dimensions in comparison with the anti-diffusion schemes. The THINC reconstruction ((16a) and (16b)) is used to compute the numerical flux which is then used in a finite volume formulation with given velocity fields as shown below. The slope parameter $\beta$ in the reconstruction function is adaptively determined in a way similar to the THINC/SW scheme [61].

Fig. 14 shows the results of a rotating circle same as Example 5.1 in [33] and Example 4.2 in [50] on grids of different resolutions. The computational domain is $[0,1] \times[0,1]$. A circle with a diameter of $1 / 4$, in which the volume fraction function is set to be 1 and 0 otherwise, is initially centered at $(5 / 8,5 / 8)$.

It is observed from Fig. 14 that the thickness of the moving interface remains compact even without the post-processing of anti-diffusion. It reveals that interface-capturing computation can be simply done by using the THINC method as a pure advection scheme. The circular geometry of the interface is preserved in the numerical solutions. The numerical results are comparable to those reported in [33] and [50].

Fig. 15 shows the result of Zalesak's problem after one revolution. The computational condition is equivalent to Example 4.3 in [50]. Again, the thickness of the jump in volume fraction remains compact throughout the computation. As we have already shown in [61], THINC method gives a comparable accuracy to the PLIC (piecewise linear interface calculation) VOF schemes [35] which are in general more accurate than the anti-diffusion type method.

We also repeat the vortex flow test as Example 5.2 in [33] and Example 4.4 in [50]. Shown in Fig. 16, the THINC scheme shows an obvious superiority in solution quality for all grid resolutions. In particular, THINC scheme is able to resolve the deformed interface with adequately accuracy even on a low resolution grid, e.g., the $32 \times 32$ grid, while the anti-diffusion schemes generate significant discrepancy not only in the shape but also in the location of the interface as shown in [33] and [50]. 
With THINC

Density

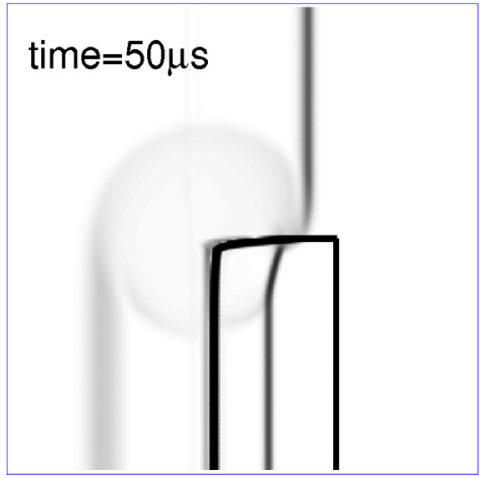

Pressure

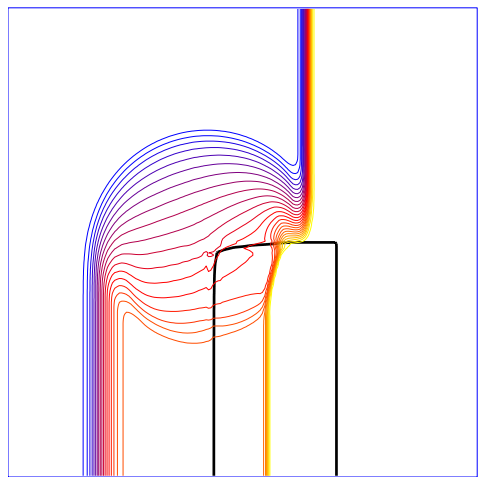

Volume fraction

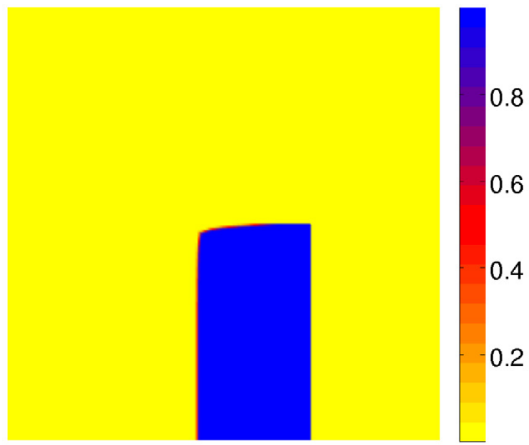

No THINC

Density

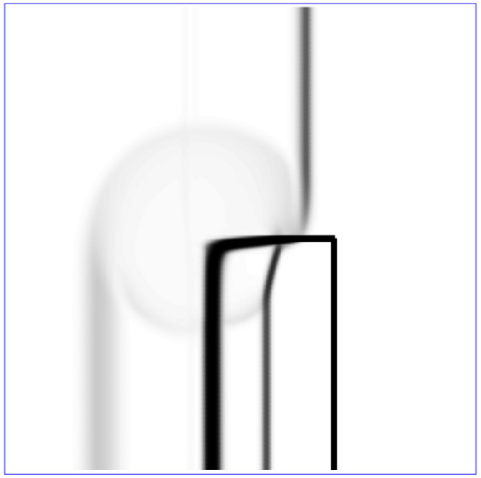

Pressure

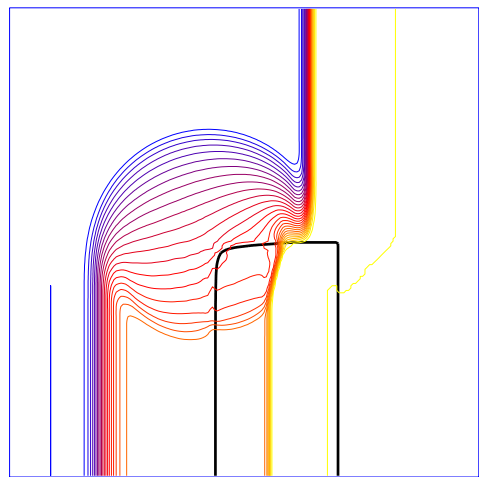

Volume fraction

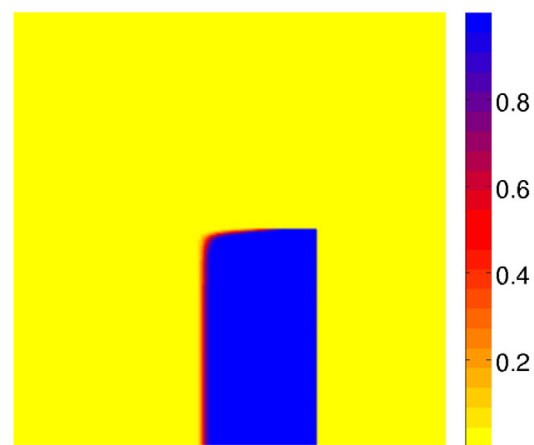

Fig. 12. Numerical results for a planar Mach 1.163 shock wave in molybdenum interacting with an encapsulated MORB liquid. Density schlieren-type images, pressure contours, and pseudo colors of volume fraction are shown at two different times $t=50$ and $100 \mu$ s obtained using each of the methods with and without THINC interface-sharpening with a $200 \times 200$ grid. In the pressure plot, the bold line represents the approximate location of the MORB-molybdenum interface.

\section{Appendix B. Anti-diffusion based interface sharpening method}

Following the previous work described in [47], an anti-diffusion based Eulerian interface-sharpening algorithm for the five-equation model (4) can be written in the form

$$
\frac{\partial q}{\partial t}+\sum_{j=1}^{N} \frac{\partial f_{j}(q)}{\partial x^{j}}+\sum_{j=1}^{N} B_{j}(q) \frac{\partial q}{\partial x^{j}}=\frac{1}{\mu} \mathcal{D}_{\varepsilon} q,
$$

where $\mathcal{D}_{\varepsilon}$ is an augmented anti-diffusion vector differential operator with a diffusion coefficient $\varepsilon$ and $\mu$ is a positive real number that the inverse of it $1 / \mu$ may be referred as the characteristic regularization rate after the work of Tiwari et 
With THINC

Density
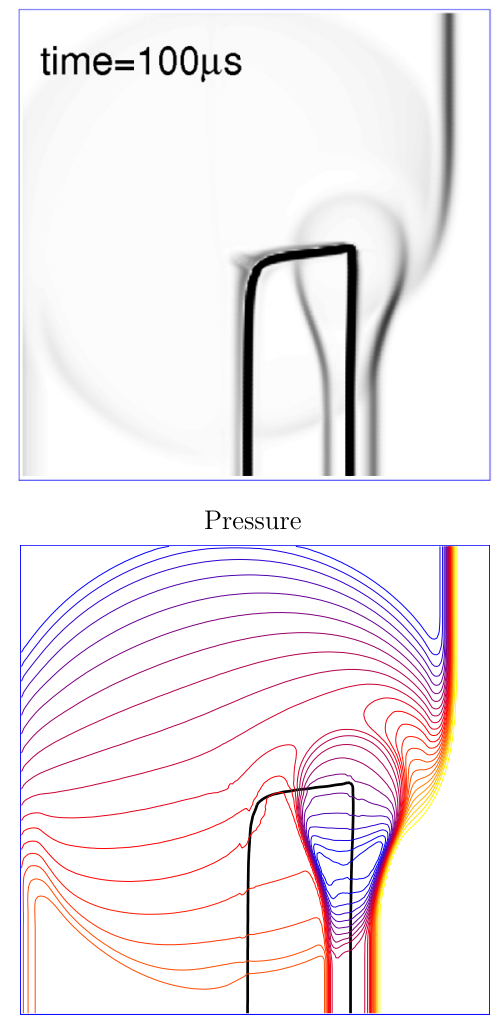

Volume fraction

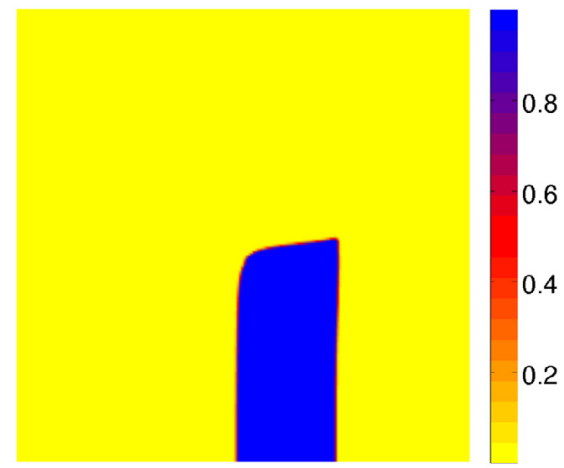

No THINC

Density
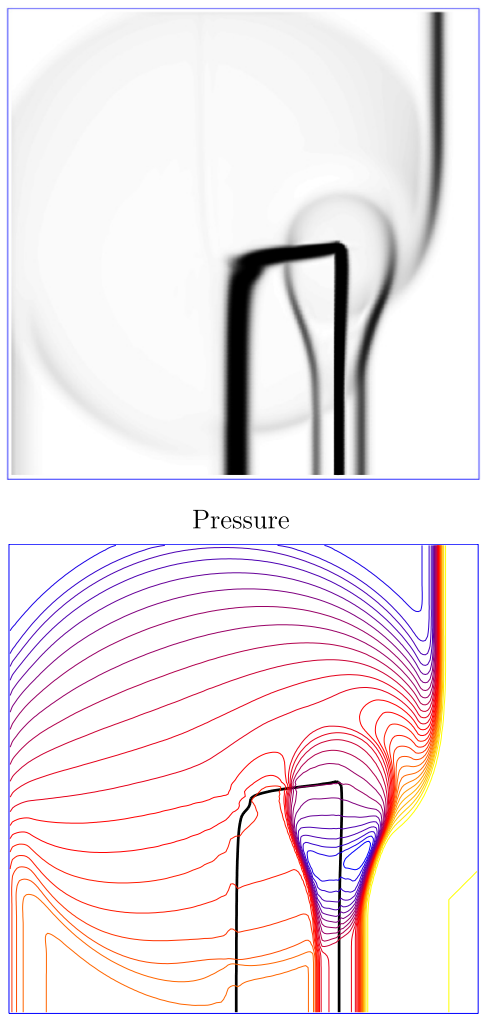

Volume fraction

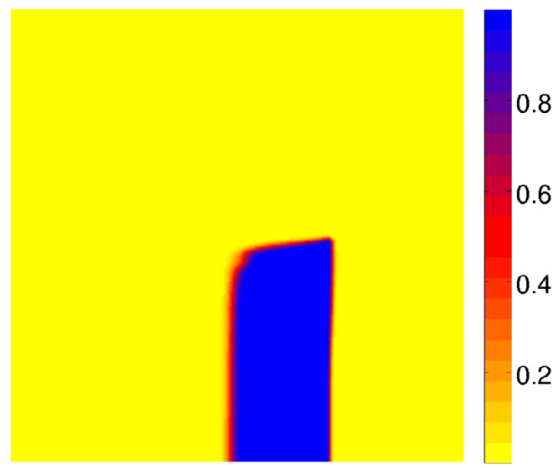

Fig. 12. (continued)

al. [54]. Here with reference to $\mathcal{D}_{\varepsilon}$ as applied to the volume fraction function $\alpha_{1}$ proposed by So et al. [50,51],

$$
\mathcal{D}_{\varepsilon} q^{(N+4)}=\mathcal{D}_{\varepsilon} \alpha_{1}=-\nabla \cdot\left(\varepsilon \nabla \alpha_{1}\right),
$$

we may define the remaining anti-diffusion terms for $\mathcal{D}_{\varepsilon} q^{(i)}, i=1,2, \ldots, N+3$ as

$$
\begin{aligned}
& \mathcal{D}_{\varepsilon} q^{(1)}=\mathcal{D}_{\varepsilon}\left(\alpha_{1} \rho_{1}\right)=\rho_{1} \mathcal{D}_{\varepsilon} \alpha_{1}, \\
& \mathcal{D}_{\varepsilon} q^{(2)}=\mathcal{D}_{\varepsilon}\left(\alpha_{2} \rho_{2}\right)=-\rho_{2} \mathcal{D}_{\varepsilon} \alpha_{1}, \\
& \mathcal{D}_{\varepsilon} q^{(j+2)}=\mathcal{D}_{\varepsilon}\left(\rho u_{j}\right)=u_{j}\left(\rho_{1}-\rho_{2}\right) \mathcal{D}_{\varepsilon} \alpha_{1}, \quad j=1,2, \ldots, N, \\
& \mathcal{D}_{\varepsilon} q^{(N+3)}=\mathcal{D}_{\varepsilon} E=\left[\frac{1}{2} \vec{u} \cdot \vec{u}\left(\rho_{1}-\rho_{2}\right)+\left(\rho_{1} e_{1}-\rho_{2} e_{2}\right)\right] \mathcal{D}_{\varepsilon} \alpha_{1},
\end{aligned}
$$




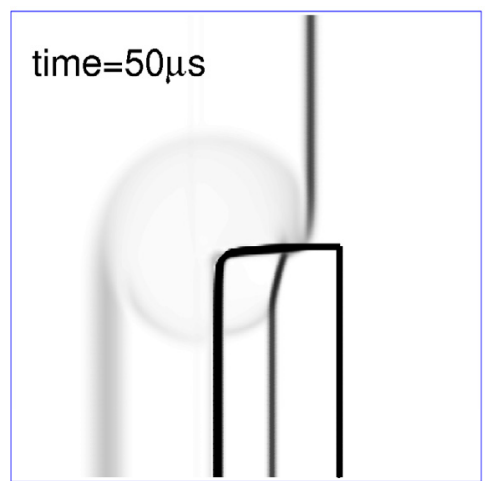

Pressure

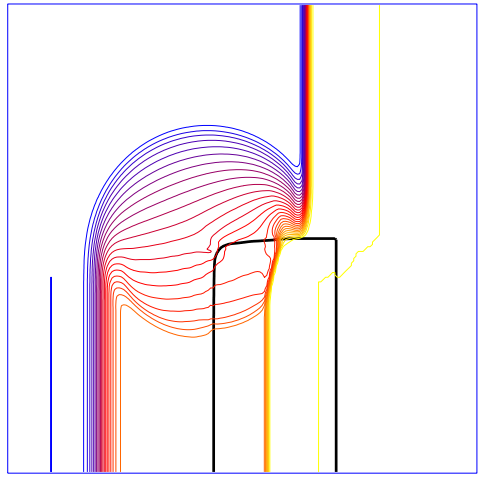

Volume fraction

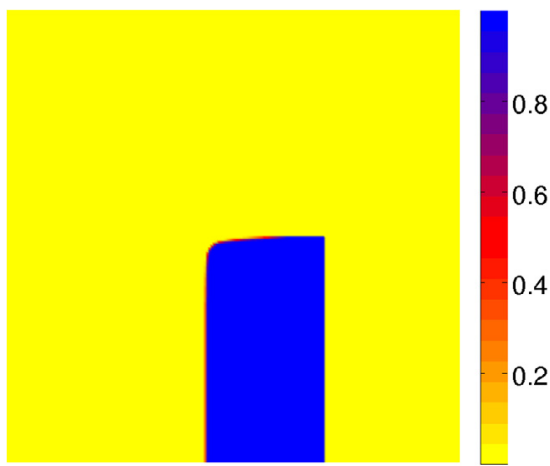

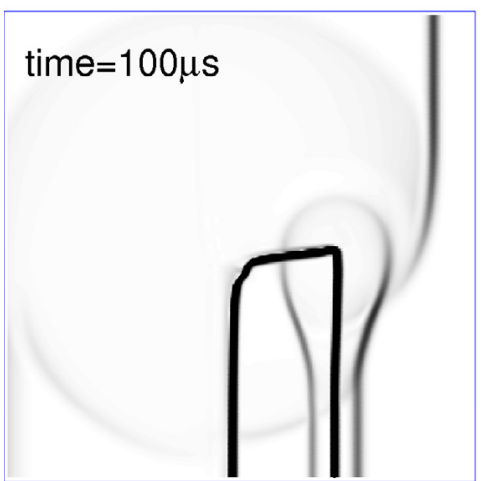

Pressure

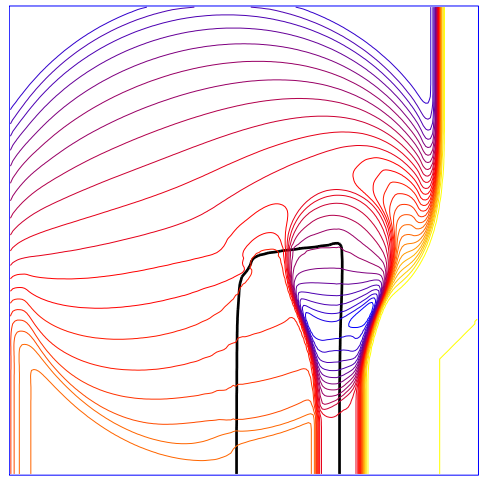

Volume fraction

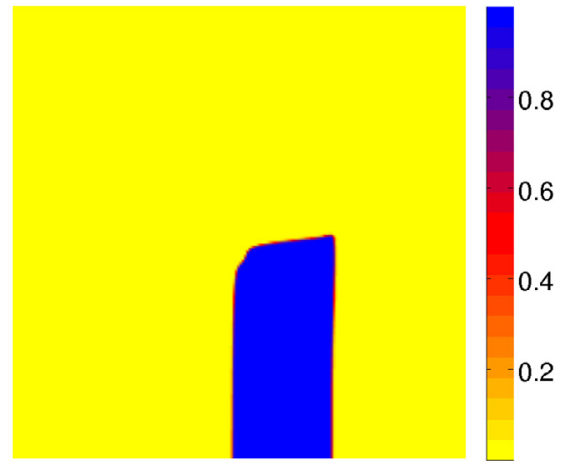

Fig. 13. Anti-diffusion results for a planar Mach 1.163 shock wave in molybdenum interacting with an encapsulated MORB liquid. Density schlieren-type images, pressure contours, and pseudo colors of volume fraction are shown at two different times $t=50$ and $100 \mu$ s with a $200 \times 200$ grid.

see [47,48,51] for the basic idea on how this can be derived, and [54] for a different view point of (B.1) if an artificial compression differential operator is use instead.

To approximate (B.1) numerically, a fractional step method that consists of the following steps in each time iteration is employed:

(1) Solve the homogeneous part of the model equation without the anti-diffusion terms,

$$
\frac{\partial q}{\partial t}+\sum_{j=1}^{N} \frac{\partial f_{j}(q)}{\partial x^{j}}+\sum_{j=1}^{N} B_{j}(q) \frac{\partial q}{\partial x^{j}}=0,
$$

over a time step $\Delta t$.

(2) Take the solution obtained in step 1 as the initial condition, and solve the model equation with only source term, 

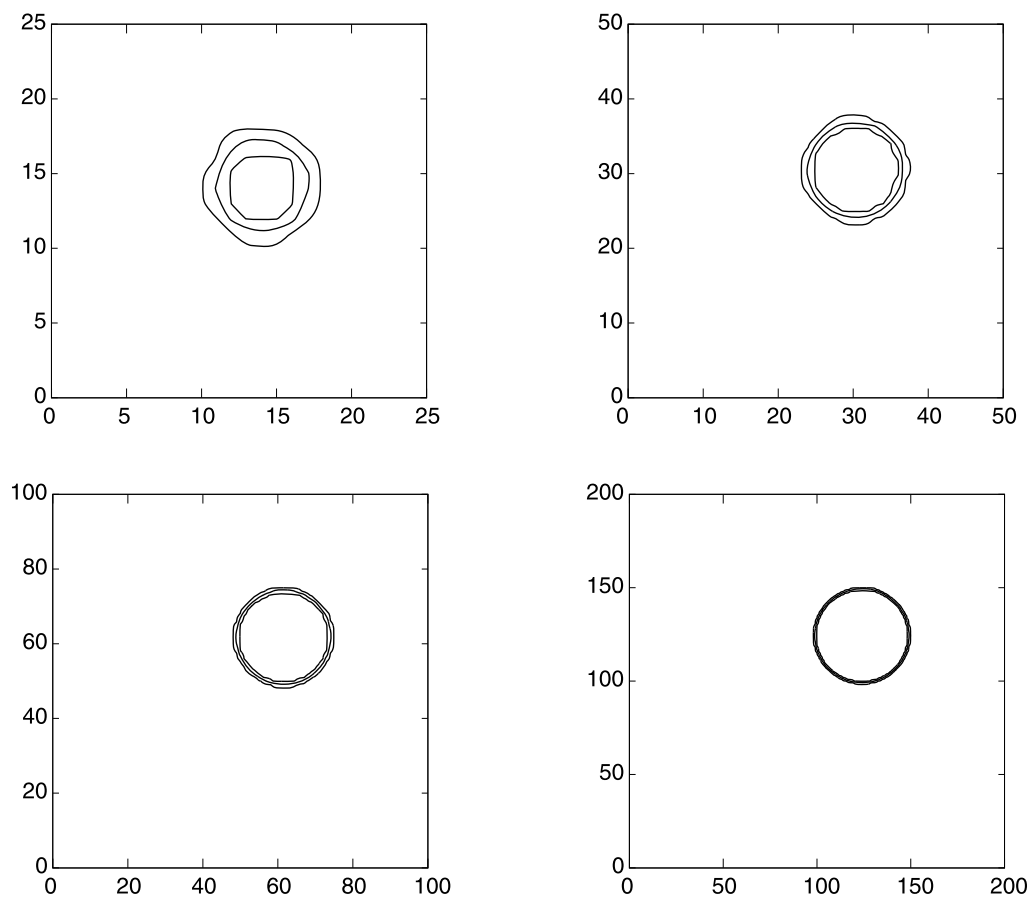

Fig. 14. The rotating circle after one revolution on grids of $25 \times 25$ (top-left), $50 \times 50$ (top-right), $100 \times 100$ (bottom-left) and $200 \times 200$ (bottom-right) respectively.
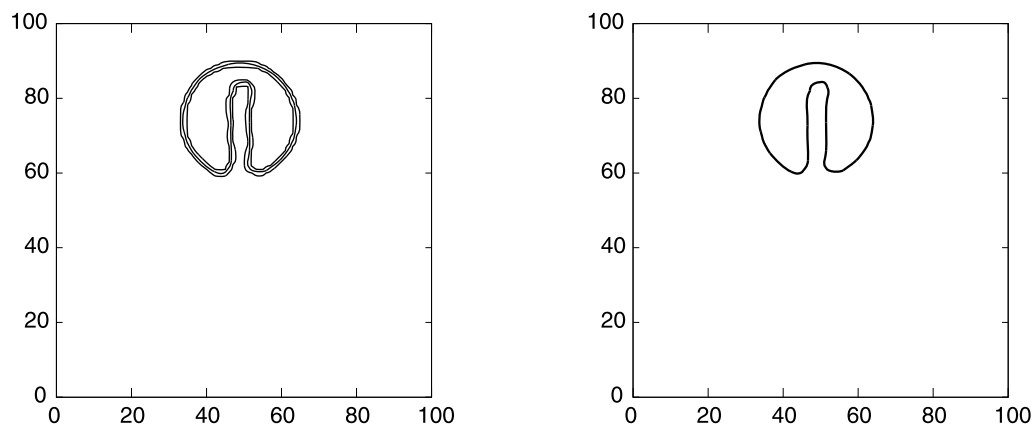

Fig. 15. The result after one revolution of Zalesak problem on a $100 \times 100$ grid. Displayed are the contours of $0.05,0.5$ and 0.95 (left) and the interface (right).

$$
\frac{\partial q}{\partial t}=\frac{1}{\mu} \mathcal{D}_{\varepsilon} q
$$

over a time step $\Delta \tau$ towards a "sharp layer". Here $\tau=t / \mu$ is a scaled time variable.

In the numerical results shown in Section 5, we have employed the standard wave propagation method in the framework of CLAWPACK [22] over a CFL-constrained $\Delta t$ in step 1, and followed by a simple explicit method based on the first-order forward Euler approximation in (pseudo) time $\tau$, and the second-order central difference approximation in space. Here for stability, in step 2 , we have taken the time step $\Delta \tau$ so that

$$
\Delta \tau \leqslant \min \left(\Delta t, \frac{\min _{j=1}^{N} \Delta\left(x^{j}\right)^{2}}{2 N \varepsilon_{\max }}\right)
$$

is satisfied, and have used a numerical regularization procedure such as employing the MinmoD limiter (cf. [20]) to stabilize the computation of $\nabla \alpha_{1}$ and so the flux $\varepsilon \nabla \alpha_{1}$ while discretizing the anti-diffusion term $D_{\varepsilon} \alpha_{1}$. Note that in the method, the diffusion coefficient $\varepsilon=\varepsilon(\vec{u})$ is a diagonal matrix with entries depending on the local velocity in both space and time. With that, we have set $\varepsilon_{\max }=\max (|\vec{u}|)$. Numerical results shown here indicate that taking only one anti-diffusion iteration is enough for the purpose of sharpening the interfaces. 

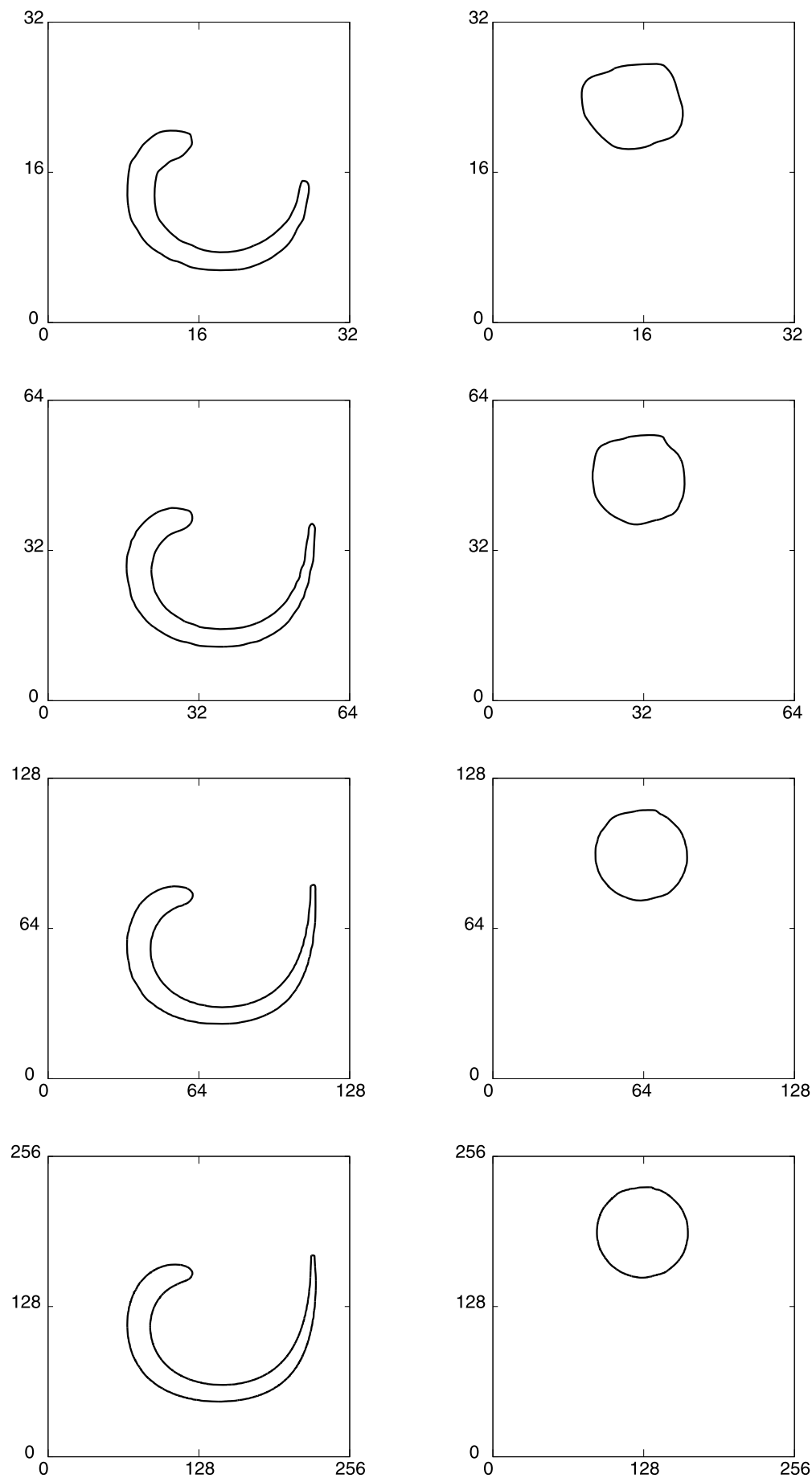

Fig. 16. The interface deformation test with a vortex shear flow on grids of different resolutions (from left to right: $32 \times 32,64 \times 64,128 \times 128$ and $256 \times 256$ respectively.). Displayed are the 0.5 -contour of the volume fraction function. The top row shows the outputs at $t=1$ and the lower row at $t=2$.

\section{References}

[1] G. Allaire, S. Clerc, S. Kokh, A five-equation model for the simulation of interface between compressible fluids, J. Comput. Phys. 181 (2002) 577-616.

[2] D.A. Cassidy, J.R. Edwards, M. Tian, An investigation of interface-sharpening schemes for multi-phase mixture flows, J. Comput. Phys. 228 (2009) 5628-5649.

[3] J. Cheng, C.-W. Shu, A high order ENO conservative Lagrangian type scheme for the compressible Euler equations, J. Comput. Phys. 227 (2007) $1567-1596$. 
[4] B. Després, F. Lagoutière, Contact discontinuity capturing schemes for linear advection and compressible gas dynamics, J. Sci. Comput. 16 (4) (2001) 479-524.

[5] J. Glimm, O.A. McBryan, R. Menikoff, D.H. Sharp, Front tracking applied to Rayleigh-Taylor instability, SIAM J. Sci. Stat. Comput. 7 (1) (1986) $230-251$.

[6] S. Gottlieb, D. Ketcheson, C.-W. Shu, Strong Stability Preserving Runge-Kutta and Multistep Time Discretizations, World Scientific, 2011.

[7] S. Gottlieb, C.-W. Shu, E. Tadmor, Strong stability preserving high-order time discretization methods, SIAM Rev. 43 (2001) $89-112$.

[8] J.-F. Haas, B. Sturtevant, Interaction of weak shock waves with cylindrical and spherical gas inhomogeneities, J. Fluid Mech. 181 (1987) 41-76.

[9] A. Harten, P.D. Lax, B. van Leer, On upstream differencing and Godunov-type schemes for hyperbolic conservation laws, SIAM Rev. 25 (1983) 35-61.

[10] R.L. Holmes, A numerical investigation of the Richtmyer-Meshkov instability using front tracking, Ph.D. thesis, SUNY at Stony Brook, August 1994 (unpublished).

[11] X.Y. Hu, B.C. Khoo, N.A. Adams, F.L. Huang, A conservative interface method for compressible flows, J. Comput. Phys. 219 (2006) $553-578$.

[12] S. Ii, K. Sugiyama, S. Takeuchi, S. Takagi, Y. Matsumoto, F. Xiao, An interface capturing method with a continuous function: the THINC method with multi-dimensional reconstruction, J. Comput. Phys. 231 (2012) 2328-2358.

[13] E. Johnsen, T. Colonius, Implementation of WENO schemes in compressible multicomponent flow problems, J. Comput. Phys. 219 (2006) 715-732.

[14] A.K. Kapila, R. Menikoff, J.B. Bdzil, S.F. Son, D.S. Stewart, Two-phase modeling of deflagration-to-denonation transition in granular materials: reduced equations, Phys. Fluids 13 (10) (2001) 3002-3024.

[15] D.I. Ketcheson, R.J. LeVeque, WENOCLAW: a higher order wave propagation method, in: Hyperbolic Problems: Theory, Numerics, Applications, SpringerVerlag, 2008, pp. 609-616.

[16] D.I. Ketcheson, K.T. Mandli, A.J. Ahmadia, A. Alghamdi, M.Q. De Luna, M. Parsani, M.G. Knepley, M. Emmett, PYCLAW: accessible, extensible, scalable tools for wave propagation problems, SIAM J. Sci. Comput. 34 (4) (2012) C210-C231.

[17] D.I. Ketcheson, M. Parsani, R.J. LeVeque, High-order wave propagation algorithms for hyperbolic systems, SIAM J. Sci. Comput. 35 (1) (2013) A351-A377.

[18] S. Kokh, F. Lagoutière, An anti-diffusive numerical scheme for the simulation of interfaces between compressible fluids by means of a five-equation model, J. Comput. Phys. 229 (2010) 2773-2809.

[19] M. Kucharik, R.V. Garimella, S.P. Schofield, M. Shashkov, A comarative study of interface reconstruction methods for multi-material ALE simulations, J. Comput. Phys. 229 (2010) 2432-2452.

[20] R.J. LeVeque, Finite Volume Methods for Hyperbolic Problems, Cambridge University Press, 2002.

[21] R.J. LeVeque, Finite Difference Methods for Ordinary and Partial Differential Equations: Steady-State and Time-Dependent Problems, SIAM, Philadelphia, 2007.

[22] R.J. LeVeque, M.J. Berger, cLAWPACK software version 4.5, http://www.clawpack.org, 2011.

[23] R.J. LeVeque, K.-M. Shyue, Two-dimensional front tracking based on high resolution wave propagation methods, J. Comput. Phys. 123 (1996) 354-368.

[24] R. Loubére, P.-H. Maire, M. Shashkov, J. Breil, S. Galera, ReALE: a reconnection-based arbitrary-Lagrangian-Eulerian method, J. Comput. Phys. 229 (2010) 4724-4761.

[25] H. Luo, J.D. Baum, R. Löhner, On the computation of multi-material flows using ALE formulation, J. Comput. Phys. 194 (2004) $304-328$.

[26] C.L. Mader, Numerical Modeling of Detonations, University of California Press, Berkeley, 1979.

[27] P.-H. Maire, R. Abgrall, J. Breil, J. Ovadia, A cell-centered Lagrangian scheme for two-dimensional compressible flow problems, SIAM J. Sci. Comput. 29 (2007) 1781-1824.

[28] A. Marquina, P. Mulet, A flux-split algorithm applied to conservative models for multicomponent compressible flows, J. Comput. Phys. 185 (2003) $120-138$.

[29] S.P. Marsh, LASL Shock Hugoniot Data, University of California Press, Berkeley, 1980.

[30] R.G. McQueen, S.P. Marsh, J.W. Taylor, J.N. Fritz, W.J. Carter, The equation of state of solids from shock wave studies, in: R. Kinslow (Ed.), High Velocity Impact Phenomena, Academic Press, San Diego, 1970, pp. 293-417.

[31] G.H. Miller, E.G. Puckett, A high order Godunov method for multiple condensed phases, J. Comput. Phys. 128 (1996) $134-164$.

[32] W.F. Noh, P. Woodward, SLIC (simple line interface calculation), in: A.I. van de Vooren, P.J. Zandbergen (Eds.), Proc. 5th Intl. Conf. on Numer. Meth. in Fluid Dynamics, Springer-Verlag, 1976.

[33] E. Olsson, G. Kreiss, A conservative level set method for two phase flow, J. Comput. Phys. 210 (2005) 225-246.

[34] S. Osher, Convergence of generalized MUSCL schemes, SIAM J. Numer. Anal. 22 (5) (1985) 947-961.

[35] J.E. Pilliod, E.G. Puckett, Second-order accurate volume-of-fluid algorithms for tracking material interfaces, J. Comput. Phys. 199 (2004) 465-502.

[36] J.W. Pritchett, An evaluation of various theoretical models for underwater explosion bubble pulsation, Technical report, IRA-TR-2-71, 1971.

[37] J.J. Quirk, S. Karni, On the dynamics of a shock-bubble interaction, J. Fluid Mech. 318 (1996) 129-163.

[38] W.J. Rider, D.B. Kothe, Reconstructing volume tracking, J. Comput. Phys. 141 (1998) 112-152.

[39] R. Saurel, R. Abgrall, A multiphase Godunov method for compressible multifluid and multiphase flows, J. Comput. Phys. 150 (1999) $425-467$.

[40] R. Saurel, F. Petitpas, R. Abgrall, Modelling phase transition in metastable liquids: application to cavitating and flashing flows, J. Fluid Mech. 607 (2008) $313-350$.

[41] C.-W. Shu, High order weighted essentially nonoscillatory schemes for convection dominated problems, SIAM Rev. 51 (2009) 82-126.

[42] R.K. Shukla, C. Pantano, J.B. Freund, An interface capturing method for the simulation of multi-phase compressible flows, J. Comput. Phys. 229 (2010) 7411-7439.

[43] K.-M. Shyue, A fluid-mixture type algorithm for compressible multicomponent flow with Mie-Grüneisen equation of state, J. Comput. Phys. 171 (2001) 678-707.

[44] K.-M. Shyue, A volume-fraction based algorithm for hybrid barotropic and non-barotropic two-fluid flow problems, Shock Waves 15 (6) (2006) 407-423.

[45] K.-M. Shyue, A wave-propagation based volume tracking method for compressible multicomponent flow in two space dimensions, J. Comput. Phys. 215 (2006) 219-244.

[46] K.-M. Shyue, A simple unified coordinates method for compressible homogeneous two-phase flows, in: E. Tadmor, J.-G. Liu, A. Tzavaras (Eds.), Proc. Symp. Appl. Math., vol. 67, American Mathematical Society, 2009, pp. 949-958.

[47] K.-M. Shyue, An anti-diffusion based Eulerian interface-sharpening algorithm for compressible two-phase flow with cavitation, in: C.-D. Ohl, E. Klaseboer, S.W. Ohl, S.W. Gong, B.C. Khoo (Eds.), Proceedings of the 8th International Symposium on Cavitation, Research Publishing Services, 2012 , p. 198.

[48] K.-M. Shyue, An Eulerian interface-sharpening algorithm for compressible gas dynamics, 2012, submitted for publication.

[49] R.W. Smith, AUSM (ALE): a geometrically conservative arbitrary Lagrangian-Eulerian flux splitting scheme, J. Comput. Phys. 150 (1999) $268-286$.

[50] K.K. So, X.Y. Hu, N.A. Adams, Anti-diffusion method for interface steepening in two-phase incompressible flow, J. Comput. Phys. 230 (2011) 5155-5177.

[51] K.K. So, X.Y. Hu, N.A. Adams, Anti-diffusion interface sharpening technique for two-phase compressible flow simulations, J. Comput. Phys. 231 (2012) 4304-4323.

[52] E. Swift, J.C. Decius, Measurement of bubble pulse phenomena, Technical report, Navord report 97-46, 1946.

[53] T. Tang, Moving mesh methods for computational fluid dynamics, in: Z.-C. Shi, Z. Chen, T. Tang, D. Yu (Eds.), Contemp. Math., vol. 383, American Mathematical Society, Rhode Island, 2005, pp. 620-625.

[54] A. Tiwari, J.B. Freund, C. Pantano, A diffuse interface model with immiscibility preservation, J. Comput. Phys. 252 (2013) 290-309.

[55] E.F. Toro, Riemann Solvers and Numerical Methods for Fluid Dynamics: A Practical Introduction, 2nd ed., Springer-Verlag, 1999. 
[56] M.A. Ullah, W. Gao, D.-K. Mao, Towards front-tracking based on conservation in two space dimensions III: Tracking interfaces, J. Comput. Phys. 242 (2013) 268-303.

[57] B. van Leer, Towards the ultimate conservative difference scheme V. A second order sequel to Godunov's method, J. Comput. Phys. 32 (1979) 101-136.

[58] A.B. Wardlaw Jr., H.U. Mair, Spherical solutions of an underwater explosion bubble, Shock Vib. 5 (1998) 89-102.

[59] M.L. Wilkins, Computer Simulation of Dynamic Phenomena, Springer, New York, 1999.

[60] F. Xiao, Y. Honma, T. Kono, A simple algebraic interface capturing scheme using hyperbolic tangent function, Int. J. Numer. Mech. Fluids 48 (2005) $1023-1040$.

[61] F. Xiao, S. Ii, C. Chen, Revisit to the THINC scheme: a simple algebraic VOF algorithm, J. Comput. Phys. 230 (2011) 7086-7092.

[62] K. Yokoi, Efficient implementation of THINC scheme: a simple and practical smoothed VOF algorithm, J. Comput. Phys. 226 (2007) $1985-2002$.

[63] A. Zein, M. Hantke, G. Warnecke, Modeling phase transition for compressible two-phase flows applied to metastable liquids, J. Comput. Phys. 229 (2010) 2964-2998.

[64] R. Zhang, M. Zhang, C.-W. Shu, On the order of accuracy and numerical performance of two classes of finite volume WENO scheme, Commun. Comput. Phys. 9 (2011) 807-827. 